\title{
Hybrid Photocatalysts Composed of Titania Modified with Plasmonic Nanoparticles and Ruthenium Complexes for Decomposition of Organic Compounds
}

\author{
Ewa Kowalska, ${ }^{\mathrm{a}, \mathrm{b},}$, Kenta Yoshiiri ${ }^{\mathrm{b}}$, Zhishun $\mathrm{Wei}^{\mathrm{a}}$, Shuaizhi Zheng ${ }^{\mathrm{c}}$, Evelyn $\mathrm{Kastl}^{\mathrm{c}}$, \\ Hynd Remita ${ }^{\mathrm{d}}$, Bunsho Ohtani ${ }^{\mathrm{a}, \mathrm{b}}$, Sven Rau ${ }^{\mathrm{c}}$ \\ ${ }^{a}$ Catalysis Research Center, Hokkaido University, N21, W10, 001-0021 Sapporo, Japan \\ ${ }^{\mathrm{b}}$ Graduate School of Environmental Science, Hokkaido University, N10, W5, 060-0810 Sapporo, Japan \\ ${ }^{c}$ Institute of Inorganic Chemistry 1, Ulm University, Albert-Einstein-Allee 11, 89081 Ulm, Germany \\ ${ }^{d}$ Laboratoire de Chimie Physique, CNRS - UMR 8000, Université de Paris-Sud, Bâtiment 349, \\ 91405 Orsay, France \\ * Corresponding author, Tel.: 81-11-706-9130; fax: 81-11-706-9133. \\ E-mail address: kowalska@ cat.hokudai.ac.jp
}

\begin{abstract}
Plasmonic photocatalysts were prepared by deposition of $2 \mathrm{wt} \%$ of gold or silver nanoparticles (NPs) on commercial titania particles with different structural properties. Ruthenium(II) complexes with phosphonic and carboxylic acid binding groups were synthesized and adsorbed on bare titania and noble metal-modified titania. The structural properties of the samples were characterized by DRS, XPS, XRD, STEM, TLC, ${ }^{1} \mathrm{H}-\mathrm{NMR}$ and 2D-COSY. Large surface area, small crystallite sizes, low pH value, nature of the deprotected phosphonate binding groups, and pre-deposited nanoparticles of noble metals enhanced the adsorption yield. Modification caused titania activation toward visible light (> $450 \mathrm{~nm}$ ) for 2-propanol oxidation and for methanol dehydrogenation under UV/vis irradiation. The modified samples exhibited enhanced activity under UV/vis irradiation for acetic acid oxidation depending on the kind of modifiers and properties of titania, i.e., 2-6, 3-9 and 1-3-fold enhancement was observed after modification with NPs of gold, NPs of silver and Ru(II) complex. The time-resolved microwave conductivity (TRMC) method proved that higher photocatalytic activity of modified titania under UV irradiation was caused by scavenging of mobile electrons by NPs of noble metals, and therefore decreasing the recombination between charge carriers. The photocatalytic activity of hybrid photocatalysts under UV/vis was influenced by the nature of the plasmonic metal and
\end{abstract}


structural properties of the metal and titania, e.g., crystallite size and polymorphic form. Different kinds of action were observed for two plasmonic metals in two reaction systems under UV irradiation, e.g., modification with an $\mathrm{Ru}(\mathrm{II})$ complex caused 12-times faster dehydrogenation of methanol for silver-modified large anatase titania (ST41) and hardly changed the activity of gold-modified samples, while during acetic acid oxidation, only hybrid photocatalysts composed of gold NPs and Ru(II) complex exhibited a slight increase of photocatalytic activity (1.1-1.3 times). The difference between gold and silver hybrid photocatalysts might have been caused by differences in surface charges of metallic deposits, i.e., the surface of silver NPs was mainly positively charged, while gold was zero-valent. Therefore, it is thought that the $\mathrm{Ru}(\mathrm{II})$ complex bound also to the surface of positively charged silver. Under visible light irradiation, fine titania modified with an $\mathrm{Ru}(\mathrm{II})$ complex exhibited the highest level of photocatalytic activity. The presence of an $\mathrm{Ru}$ (II) complex highly enhanced photocatalytic activity of titania modified with plasmonic NPs. However, NPs of plasmonic metals hindered the photoactivity of $\mathrm{Ru}(\mathrm{II})$-titania.

\section{Keywords:}

Hybrid photocatalysts, $\mathrm{Ru}(\mathrm{II})$ complexes, plasmonic photocatalyst, heterogeneous-homogeneous photocatalysis, gold nanoparticles, silver nanoparticles, LSPR

\section{Introduction}

Though semiconductor photocatalysis has become more and more commonly used in recent years, a wide range of applications is still limited for the following reasons: i) fast recombination of charge carriers $\left(\mathrm{e}^{-} / \mathrm{h}^{+}\right)$, which is typical for all semiconducting materials, ii) absorption below $400 \mathrm{~nm}$ by the most active photocatalysts, e.g., $\mathrm{TiO}_{2}$, and iii) impossibility of water splitting by visible light-absorbing semiconductors with a narrow bandgap [1-3]. Among the various available methods, surface modification with noble metals seems advisable due to acceleration of photoexited electron transfer to the substrate [4-7] and ability of visible light absorption either by metal complexes bounded to the semiconductor [8] or by photoexcitation of localized surface plasmon resonance (LSPR) of nanoparticles (NPs) of noble metals attached to the semiconductor [9].

In contrast to catalytically active gold NPs, where nano-sized gold has been recommended for a high level of activity [10], our results showed that polydispersity in the size and shape of deposited gold NPs on titania was beneficial for photocatalytic 
activity under visible light irradiation [11-13]. It was proposed that a wide size/shape distribution of gold NPs, and thus the ability of photoabsorption of a broad range of wavelengths, was responsible for the high level of photoacatalytic activity.

Though desirable photoabsorption properties of plasmonic photocatalysts can be easily obtained, e.g., by preparation of NPs with different sizes and shapes [14-16], their photocatalytic activities under visible light irradiation are still low and should be improved. In this regard, we have proposed hybrid photocatalysts composed of plasmonic NPs, titania and ruthenium complexes, as potential efficient materials for decomposition of organic compounds under solar radiation.

Ruthenium complexes can sensitize titania toward visible light as was proposed by O'Regan and Graetzel [17], and since then they have been extensively examined for application in dye-sensitized solar cells (DSSCs) [18, 19]. The large absorption cross section in the visible range populating a highly reactive MLCT state makes this type of polypyridyl $\mathrm{Ru}(\mathrm{II})$ complex very interesting $[20,21]$. The relatively long-lived exited states of ruthenium complexes allow efficient electron injection from Ru chromophores into the conduction band (CB) of titania upon light excitation. In order to ensure efficient charge injection into titania, functional groups such as carboxylate and phosphonate derivatives have been utilized to bind the $\mathrm{Ru}(\mathrm{II})$ complex to the surface of the semiconductor. An essential point is the aqueous stability of the resultant surface attachment, which is important for commercial application [22]. It has been reported that phosphonate derivatives are more stable than carboxylate in an aqueous environment [23].

\section{Experimental}

\subsection{Preparation of photocatalysts}

Homogeneous, heterogeneous and hybrid photocatalysts were prepared by inorganic synthesis, deposition of metal NPs on titania, and combining homogeneous and heterogeneous photocatalysts by mixing, respectively, as is briefly described in the following sections.

\subsubsection{Preparation of titania modified with plasmonic NPs}

Plasmonic photocatalysts were prepared by photodeposition of gold or silver NPs (2 $\mathrm{wt} \%$ ) on commercial titania particles [12]. Chloroauric acid and silver nitrate were used as metal sources. $\mathrm{TiO}_{2}$ samples were suspended in $50 \mathrm{vol} \%$ aqueous methanol to which an aqueous solution of the metal precursor was added and then oxygen was removed by argon bubbling (at least $15 \mathrm{~min}$ ). The suspensions were UV-irradiated for 
$1 \mathrm{~h}$ at $298 \mathrm{~K}$ with magnetic stirring $(500 \mathrm{rpm})$. Liberated hydrogen from methanol suspensions was measured by gas chromatography (GC-TCD). After photodeposition, samples were washed at least three times with methanol and a few times with Milli-Q water, dried at $393 \mathrm{~K}$, and ground in an agate mortar.

\subsubsection{Synthesis of ruthenium complexes}

Two types of tris(bipyridine) ruthenium(II) complexes were synthesized with carboxylate $(\mathrm{Ru}(\mathrm{II}) \mathrm{Carb})$ or phosphonate $(\mathrm{Ru}(\mathrm{II}) \mathrm{Phos})$ substituents attached to the bipyridine group at $4,4^{\prime}$ positions. The ruthenium(II) carboxylate complex $\left[(\text { bpy })_{2} \mathrm{Ru}\left(\mathrm{bpy}(\mathrm{COOH})_{2}\right)\right]\left[\mathrm{PF}_{6}\right]_{2}$ (Ru(II)Carb) was prepared according to literature method [24]. Two complexes featuring ester-protected phosphonate substituents were synthesized according to methods used by Gillaizeau-Gauthier et al. [25] and Zabri et al. [26]. The first product of the complexation reaction of bpdp(OEt) ${ }_{4} \quad\left(4,4^{\prime}-\right.$ bis(ethylester)phosphonate)-2,2'-bipyridine) with $\quad \mathrm{Ru}(\text { bpy })_{2} \mathrm{Cl}_{2} \quad$ was $\left[(\mathrm{bpy})_{2} \mathrm{Ru}\left(\mathrm{bpdpH} \mathrm{H}_{2}(\mathrm{OEt})_{2}\right)\right] \mathrm{Cl}_{2}$ in which one ester group on each phosphonate was hydrolized to an $\mathrm{OH}$-group (half deprotected, $\mathrm{Ru}(\mathrm{II}) \_1$ ). From this substance, it was possible to either reprotect the $\mathrm{OH}$-groups using a standard procedure (oxalyl chlorid and ethanole) to obtain $\left[(\text { bpy })_{2} \mathrm{Ru}\left(\operatorname{bpdp}(\mathrm{OEt})_{4}\right)\right] \mathrm{Cl}_{2}$ (protected, $\left.\mathrm{Ru}(\mathrm{II}){ }_{-} 2\right)$ [26] or remove the remaining two ester groups to obtain $\left[(\mathrm{bpy})_{2} \mathrm{Ru}\left(\mathrm{bpdpH} \mathrm{H}_{4}\right)\right] \mathrm{Cl}_{2}$ with four $\mathrm{OH}$-groups available (fully deprotected, $\mathrm{Ru}(\mathrm{II})$ ). This last step was done by a new procedure using literature known reactions [26, 27], resulting in only 95\% maximum grade (calculated by comparing integrals in ${ }^{1} \mathrm{H}-\mathrm{NMR}$ ). Therefore, cleavage of the ester bond was performed under basic conditions using $\mathrm{NaOH}(\mathrm{aq})$ which produced $100 \%$ deprotection of the phosphonate groups. Details of synthesis and characterization of the obtained products are shown in Supplementary Information (s1, and Fig. s1).

\subsubsection{Preparation of hybrid photocatalysts}

Hybrid photocatalysts were obtained by adsorption of the ruthenium complexes on five bare or metal-modified titania photocatalysts $(0.16-0.32 \mathrm{~mol} \%)$ by mixing. Nineteen $\mathrm{mg} \mathrm{Ru}(\mathrm{II}) \mathrm{Carb}$ and $50 \mathrm{mg} \mathrm{Ru}(\mathrm{II}) \mathrm{Phos}$ (of each complex: Ru(II), Ru(II)_1, Ru(II)_2) were dissolved in $100 \mathrm{ml}$ acetonitrile and water, respectively. Then $1 \mathrm{~g} \mathrm{TiO}_{2}$ was added, suspended by 5-min ultrasonication, and stirred with a magnetic stirrer. During stirring, $1.5 \mathrm{~mL}$ of the suspension was collected and centrifuged. The supernatant was diluted $(20 \mathrm{x})$ with water and UV/vis analyzed to check the amount of non-adsorbed complex. 


\subsection{Characterization of photocatalysts}

Homogeneous, heterogeneous and hybrid photocatalysts were characterized by various methods, e.g., by UV/vis spectroscopy, diffuse reflectance spectroscopy (DRS), X-ray crystallography (XRD), scanning transmission electron microscopy (STEM), X-ray photoelectron spectroscopy (XPS), thin layer chromatography (TLC), proton nuclear magnetic resonance spectroscopy $\left({ }^{1} \mathrm{H}-\mathrm{NMR}\right)$, and two-dimensional homonuclear correlation spectroscopy (2D-COSY). Details of the equipment and operation parameters are shown in Table 1.

The crystallinity of titania was evaluated by the internal standard method, where $20 \mathrm{wt} \%$ highly crystalline nickel oxide (NiO, the standard) was mixed thoroughly with a sample ( $80 \mathrm{wt} \%$ ) by braying in an agate mortar.

Specific surface area of the photocatalysts were estimated by nitrogen adsorption at $77 \mathrm{~K}$ using the Brunauer-Emmett-Teller (BET) method.

The charge-carrier lifetimes in $\mathrm{TiO}_{2}$ after UV illumination were determined by microwave absorption experiments using the time-resolved microwave conductivity (TRMC) method [28].

Table 1

Details of experimental equipment and operation parameters used for sample characterization

\begin{tabular}{ll}
\hline Method & Equipment type and operation parameters \\
\hline UV/vis & Varian Cary 50; 200-800 nm \\
BET & Yuasa-Quantachrome NOVA 1200e surface and pore analyzer \\
DRS & JASCO V-670 equipped with a PIN-757 integrating sphere; Poly(tetrafluoroethylene) \\
& reference, barium sulfate and bare titania used as reference \\
XRD & Rigaku Intelligent XRD system, SmartLab equipped with a sealed tube X-ray generator \\
& (a copper target, 40 kV, 30 mA), a D/teX high-speed position-sensitive detector system \\
& and an ASC-10 automatic sample changer; 20 range: $10-90^{\circ}$, scan speed: $1.00^{\circ} \mathrm{min}^{-1}$, \\
& scan step: 0.008 $;$ XRD patterns analyzed by Rigaku PDXL; crystal structure analysis \\
& package including Rietveld analysis (RIETAN FP [29]) \\
STEM & HITACHI HD2000 ultrathin evaluation system (200 kV accelerating voltage, $30 \mu \mathrm{A}$ \\
& emission current). Powders were dispersed in ethanol in an ultrasonic bath for a few \\
& minutes, and suspension droplets were deposited on a carbon-covered copper microgrid \\
& (Onken, types A and B). Images were acquired at a wide range of magnifications \\
& (200,000-800,000) in normal resolution modes (working distance: 3 mm) as secondary \\
& electron (SE), Z-contrast (ZC), and bright-field (BF) modes \\
& JEOL JPC-9010MC, MgK $\alpha$ X-ray \\
\hline
\end{tabular}


${ }^{1} \mathrm{H}-\mathrm{NMR} \quad$ JEOL $270 \mathrm{MHz} ; 270 \mathrm{MHz}$ in $\mathrm{CD}_{3} \mathrm{CN}, \mathrm{MeOD}$ and $\mathrm{D}_{2} \mathrm{O}$

2D-COSY JEOL $400 \mathrm{MHz} ; 400 \mathrm{MHz}$ in $\mathrm{MeOD}$ and $\mathrm{D}_{2} \mathrm{O}$

\subsection{Photocatalytic activity measurements}

Photocatalytic activities for five titania (listed in Table 2) single-modified, i.e., with plasmonic metals and deprotected $\mathrm{Ru}(\mathrm{II})$ complex of $\left[(\mathrm{bpy})_{2} \mathrm{Ru}\left(\mathrm{bpdpH} \mathrm{H}_{4}\right)\right] \mathrm{Cl}_{2}(\mathrm{Ru}(\mathrm{II}))$, and double-modified photocatalysts, i.e., hybrid photocatalysts, were tested in the following three reaction systems:

i) oxidation of acetic acid under UV/vis irradiation,

ii) dehydrogenation of methanol under UV/vis irradiation,

iii) oxidation of 2-propanol under visible light irradiation $(\lambda>450 \mathrm{~nm})$.

Generally, $10 \mathrm{mg}$ of a photocatalyst was suspended in $5 \mathrm{~mL}$ of aqueous solutions of acetic acid (5 vol\%), 2-propanol (5 vol\%) or methanol (50 vol\%) and photoirradiated under magnetic stirring in a thermostated water bath at $298 \pm 5 \mathrm{~K}$. UV/vis and visible experiments were carried out with a $400 \mathrm{~W}$ high-pressure mercury lamp and $300 \mathrm{~W}$ xenon lamp, respectively. The emission spectra of both light sources and details of experimental systems were described in detail in previous reports [12, 30]. For testing dehydrogenation of methanol, air was removed from the reaction tube by argon bubbling for at least $15 \mathrm{~min}$.

The reaction products, carbon dioxide, hydrogen and acetone, were analyzed by gas chromatography, i.e., GC-TCD (Shimadzu GC8A-IT) and GC-FID (Shimadzu GC-14B).

For action spectra analyses, $20 \mathrm{mg}$ of a photocatalyst was suspended in $2 \mathrm{~mL}$ of aqueous acetic acid $(5 \mathrm{vol} \%)$ and irradiated for $1 \mathrm{~h}$ by a diffraction grating type illuminator equipped with a $300 \mathrm{~W}$ xenon lamp (Jasco CRM-FD and Hamamatsu Photonics C2578-02, respectively). The intensity of irradiation, measured by a Hioki 3664 optical power meter, was in the range of $1-4 \times 10^{-8}$ einstein $\mathrm{s}^{-1}$. During the irradiation, $0.2 \mathrm{~mL}$ gas phase of the reaction mixture was withdrawn with a syringe and injected into a gas chromatograph for carbon dioxide $\left(\mathrm{CO}_{2}\right)$ analysis (Shimadzu GC-14B, equipped with a flame ionization detector and a methanizer (Shimadzu MTN-1)).

\section{Results and discussion}

\subsection{Binding of Ru complexes to titania}

\subsubsection{Ru complexes with carboxylic anchoring groups (Ru(II)Carb)}

$\mathrm{Ru}(\mathrm{II}) \mathrm{Carb}$ (dissolved in acetonitrile) was adsorbed efficiently (>95\%) and durably on 
titania P25, since even extended mixing $(60 \mathrm{~h})$ did not cause its desorption in pure acetonitrile, as shown in Fig. 1. However, addition of water (sample washing after preparation) resulted in complete detachment of the complex from titania, due to hydrolytic cleavage, as has already been reported [31]. For practical application, stable binding in an aqueous environmental is essential, and adsorption of detached $\mathrm{Ru}(\mathrm{II})$ Carb was thus continued for fresh titania ST01 $(1 \mathrm{~g})$ at various pH values (Fig. s2). Only 5\% and $9 \%$ of the complex bound to titania at $\mathrm{pH}$ values of 5.6 and 3.5, respectively. Basic conditions $(\mathrm{pH}=9.4)$ resulted in quick but reversible complex desorption, i.e., change in the $\mathrm{pH}$ value to $7-9.4$ and then to 3.2 resulted in $4.5 \%$ and $18 \%$ complex adsorption, respectively.

Enhanced adsorption at a low $\mathrm{pH}$ value can be explained by electrostatic interactions between the negatively charged sensitizer molecule and positively charged $\mathrm{TiO}_{2}$ surface, while the negatively charged titania surface under basic conditions repeled dye molecules [32]. Long-lasting stirring (ca. 100 days) did not cause complex desorption, as shown in Fig. s2. Therefore, it has been found that an Ru complex with carboxylic anchoring groups can be partly adsorbed $(<20 \%)$ on titania in an aqueous environment with high stability under acidic conditions.

Fig. 1. $\mathrm{Ru}(\mathrm{II})$ Carb adsorption on titania P25 in acetonitrile (0.16 mol\%): left) UV/vis absorbance spectra of the supernatant for $0,1.5,3.5,7,23,25,28$ and $60 \mathrm{~h}$ of adsorption; right) Adsorption efficiency; inset) Chemical structure of Ru(II)Carb.

\subsection{2. $R u$ complexes with phosphonate anchoring groups ( $R u(I I) P h o s)$}

Three kinds of phosphonate anchoring groups, i.e., protected (Ru(II)_2), half-protected $\left(\mathrm{Ru}(\mathrm{II}) \_1\right)$ and deprotected ( $\mathrm{Ru}(\mathrm{II})$, were investigated. At first, $\mathrm{Ru}(\mathrm{II}) \mathrm{Phos}$ complexes with different anchoring groups were adsorbed on two kinds of titania: fine $(8 \mathrm{~nm})$ anatase (ST01) and large $(517 \mathrm{~nm})$ rutile (Ald_R). As was expected, Ru(II) complex with deprotected phosphonate groups showed much better adsorption on titania than protected ones (Ru(II)_2) as shown in Fig. 2. However, the kind of titania influenced adsorption yield even more strongly than did anchoring groups, and thus adsorption of $\mathrm{Ru}(\mathrm{II}) \mathrm{Phos}$ on rutile NPs was negligible independently of the anchoring groups. It was reported that a much smaller amount of free adsorption sites was observed for a dye-sensitized film composed of fine titania NPs $(20 \mathrm{~nm})$ than for a film composed of large titania NPs (300 nm) [33].

Fig 2. Adsorption efficiency of $\mathrm{Ru}(\mathrm{II}) \mathrm{Phos}$ on anatase (ST01, closed symbols) and rutile 
(Ald_R), open symbols); $\diamond, \diamond-\mathrm{Ru}(\mathrm{II}), \mathbf{\square}, \square-\mathrm{Ru}(\mathrm{II}) \_1, \boldsymbol{\Delta}, \Delta-\mathrm{Ru}(\mathrm{II}) \_2$.

More detailed analysis was performed to further investigate this effect. Since binding probability may be influenced by structural properties of titania as well as the conditions of titania modification, several test systems were investigated. $\mathrm{Ru}$ (II) complexes were adsorbed on eleven commercial titanias with different structural properties (details shown in Table s1). It was found that not polymorphic form but small crystallite size and large surface area caused efficient adsorption on titania, as shown in Figs. 3 and 4.

Fig. 3. Representative UV/vis absorbance spectra of the supernatant during Ru(II) adsorption on anatase titania: ST01 (left) and ST41 (right).

Fig. 4. left) Effects of crystallite size $(\boldsymbol{\Lambda}, \Delta)$ and specific surface area $(\bullet, \circ)$ of titania on efficiency of $\mathrm{Ru}(\mathrm{II})$ adsorption after $2 \mathrm{~h}$ of mixing: anatase (closed symbols) and rutile (open symbols) titania; inset: STEM image of bare rutile (Ald_R); right) STEM image of bare anatase (ST01).

A low $\mathrm{pH}$ value enhanced binding yield, similarly to $\mathrm{Ru}(\mathrm{II}) \mathrm{Carb}$ adsorption. The impact of $\mathrm{pH}$ depended also on titania properties, and it was found that adsorption of $\mathrm{Ru}(\mathrm{II})$ on fine anatase (ST01) proceeded almost the same at $\mathrm{pH} 3$ and $\mathrm{pH} 7$ and proceeded much slower under basic conditions $(\mathrm{pH}=10)$ reaching ca. $80 \%$ and $20 \%$ after 15 min of mixing (Fig. 5, left). However, adsorption on large rutile (Ald_R) proceeded much slower and was highly dependent on $\mathrm{pH}$, reaching 2, 18 and $31 \%$ adsorption at $\mathrm{pH}$ 10, 7 and 3, respectively, after $1 \mathrm{~h}$ of stirring (Fig. 5, right). Detailed investigation of the factors governing surface binding of ruthenium complexes yielded important materials chemistry factors for visible light-driven photocatalytic systems for water oxidation [34].

Fig. 5. Influence of pH on adsorption efficiency of Ru(II) on titania ST01 (left) and Ald_R (right).

For the development of noble metal-containing hybrid photocatalysts, it is of great interest whether the presence of pre-deposited NPs of noble metals influence binding of the ruthenium complexes. A relatively complicated behavior was evident from our results. No significant influence was observed for the first two hours of 
adsorption. However, the presence of noble metals, especially silver, enhanced Ru(II) binding during adsorption for a long time, as shown in Fig. 6. At present, it is difficult to discuss such behavior, and more studies using other titania samples are currently being performed. It is proposed that long-lasting stirring caused aggregation of noble metals and detachment of the noble metals from the titania surface, as was previously observed [35]. Consequently, Ru(II) complex could be adsorbed much more easily at titania active sites after detachment of NPs (NPs of noble metals being mainly generated on surface defects [36]). Another possible explanation is the differences in surface charges of noble metal NPs, i.e., it is possible that $\mathrm{Ru}$ (II) firstly adsorbed on the titania surface and then on NPs of noble metals at a higher rate in the case of positively charged silver than zero-valent gold (XPS data of surface charges will be discussed later.).

Fig. 6. Influence of pre-deposited metals on $\mathrm{Ru}(\mathrm{II})$ binding efficiency during long (left) and short (right) time adsorption.

All photocatalysts showed high stability during stirring for a long time. There seems to be a rather inconsistent data set in the current literature with regard to this question, i.e., observed stability and instability of $\mathrm{Ru}(\mathrm{II})-\mathrm{TiO}_{2}$ even in the dark $[22,23]$.

\subsection{Structural properties of hybrid photocatalysts}

Hybrid photocatalysts were prepared on five bare and metal-modified titania photocatalysts: STG1, ST41, Merck, P25 and TIO10. The sizes of crystallites and content of crystal phases were determined by XRD analysis. The XRD patterns are shown in Figs. 7 and s3, and XRD data are summarized in Table 2. Three photocatalysts, ST41, Merck and TIO10, consisted of 98\%, 93\% and 86\% of anatase phase, respectively, and $1 \%, 4 \%$ and $0 \%$ of rutile phase, respectively. Titania P25, which is well known for its high level of photocatalytic activity, consisted of $82 \%, 15 \%$ and $3 \%$ of anatase, rutile and non-crystaline phases, respectively. It must be pointed that P25 is highly heterogeneous, and even samples taken from the same bottle can differ in their composition, i.e., contents of anatase, rutile and non-crystalline phases varied between $73 \%$ and $85 \%, 14 \%$ and $16 \%$ and $0 \%$ and $13 \%$, respectively [37]. One sample, STG1, consisted of almost only rutile phase (97\%).

The sizes of metallic crystallites were smallest and largest for the smallest and largest particles of titania, as was reported for gold-modified titania [12]. It has been proposed that metal NPs were mainly formed on crystalline defects [36] and that fine 
particles of titania possessing a large amount of such defects [38] induced formation of fine metallic NPs. On the other hand, large metallic NPs were formed on the surface of well-crystallized rutile titania of large NPs (STG1).

Fig. 7. XRD patterns of bare, silver-modified and gold-modified titania: top) rutile STG1, bottom) anatase ST41; insets) enlargement showing (111) and (200) facets of gold and silver.

Plasmonic and hybrid photocatalysts exhibited a broad range of photoabsorption from 200 to $800 \mathrm{~nm}$ as shown in Table 2, Fig. 8. Absorption in the UV range was caused by the titania semiconducting structure with bandgaps of ca. 3.2 and $3.0 \mathrm{eV}$ for anatase and rutile polymorphic forms, respectively. On the other hand, absorption in the visible range resulted from localized surface plasmon resonance (LSPR) of gold/silver NPs and metal to ligand charge transfer (MLCT) of the Ru complex. MLCT absorption was much narrower $(400-500 \mathrm{~nm})$ than that by plasmonic metals (400-800 nm), since NPs of different sizes and shapes were obtained by photodeposition, and thus with broad LSPR [12]. Consequently, hybrid photocatalysts, having MLCT and LSPR, showed enhanced photoabsorption in the whole tested range, i.e., $200-800 \mathrm{~nm}$.

Fig. 8. Absorption spectra (DRS) of bare and modified titania: (a) STG1, (b) P25, (c) Merck, d) ST41 and (e) TIO10.

Table 2

Properties of hybrid photocatalysts

\begin{tabular}{llllllllll}
\hline & BET & \multicolumn{2}{c}{ Crystalline size/nm } & \multicolumn{2}{c}{ LSPR/nm } & \multicolumn{3}{c}{$\mathrm{TiO}_{2}$ composition (\%) } \\
& $/ \mathrm{m}^{2} \mathrm{~g}^{-1}$ & $\mathrm{TiO}_{2}$ & $\mathrm{Au}$ & $\mathrm{Ag}$ & $\mathrm{Au}$ & $\mathrm{Ag}$ & anatase & rutile & non-crystal \\
\hline ST41 & 10.8 & $208^{*}$ & 29.2 & 33.8 & 570 & 532 & 98.2 & 0.7 & 1.1 \\
Merck & 11.1 & $169^{*}$ & 30.3 & 12.0 & 571 & 505 & 93.2 & 4.5 & 2.4 \\
STG1 & 5.9 & $250^{*}$ & $43.0^{*}$ & 34.6 & 576 & 524 & 0.2 & 96.8 & 2.9 \\
P25 & 57.4 & $25^{\mathrm{A}}$, & 17.0 & 19.8 & 553 & 595 & 81.7 & 15.4 & 3.0 \\
& & $40^{\mathrm{R}}$ & & & & & & & \\
TIO10 & 334.8 & $15^{*}$ & 9.4 & 2.5 & 559 & & 86.2 & 0 & 13.8 \\
\hline
\end{tabular}

* values determined and reported previously $[12,39],{ }^{\mathrm{A}}$ anatase, ${ }^{\mathrm{R}}$ rutile.

Atomic composition and chemical characteristics of elements incorporated in the surface layer of modified titania were investigated by XPS analysis. The presence of silver and gold was confirmed in all examined samples. The amount of silver 
highly exceeded that which was used for deposition (2 wt $\%$ ), reaching, for example, 8 and $10 \mathrm{wt} \%$ for single-modified and double-modified titania P25. The amounts of gold reached $1.9 \mathrm{wt} \%$ and $2 \mathrm{wt} \%$ for $\mathrm{Au}-\mathrm{P} 25$ and $\mathrm{Au} / \mathrm{Ru}(\mathrm{II})-\mathrm{P} 25$, respectively. Since the mean sizes of gold and silver NPs determined by XRD were almost the same as the size of anatase crystallite, uniform distribution of metallic NPs in the bulk was expected. Therefore, a much larger amount of silver NPs on the surface indicated the presence of very fine (nano-sized) NPs of silver undetectable by XRD (For confirmation, HR-TEM investigation will be performed.). In the case of large anatase particles (ST41), a similar tendency was observed, i.e., a much larger amount of silver than that of gold on the surface, reaching $15 \mathrm{wt} \%$ and $3 \mathrm{wt} \%$, respectively. The results obtained for the surface enriched with metallic NPs were expected since these samples possessed very large titania crystallites on which metallic deposits were mainly formed on the surface (not uniformly mixed with titania $\mathrm{NP}_{\mathrm{S}}$ in the bulk). Smaller sizes of silver NPs than gold NPs and existence of nano-sized metals were confirmed by STEM observations, as shown in Figs. 9 and s4 (higher magnification images showing nano-sized gold and silver).

Fig. 9. STEM images of silver-modified (left) and gold-modified (right) titania STG1 (top) and ST41 (bottom).

XPS analysis of Au4f and $\mathrm{Ag} 3 \mathrm{~d}$ after deconvolution indicated that two or three components could be detected, i.e., $\mathrm{Au}^{8+}, \mathrm{Au}^{0}$ and $\mathrm{Au}^{8-}$ for $\mathrm{BE}$ of 83.6, 82.8 and $81.8 \mathrm{eV}$, respectively, and $\mathrm{Ag}^{0}, \mathrm{Ag}^{+}$and $\mathrm{Ag}^{2+}$ for $\mathrm{BE}$ of 368.4, 367.4 and 366.3, respectively. Surface silver existed predominantly in the positively charged form (79-88\%), while gold was mainly zero-valent (83-97\%), as shown for titania P25 in Fig. 10. Binding of $\mathrm{Ru}$ (II) complex on the surface of titania modified with plasmonic NPs only slightly changed charges on the surface of metallic NPs.

Fig. 10. XPS data of titania P25 modified with silver, gold and $R u(I I)$; representative XPS spectra for P25 modified with A) Ag/Ru(II) and B) Au.

\subsection{Photocatalytic activities of a hybrid photocatalyst}

\subsubsection{Photocatalytic decomposition of acetic acid under UV/vis irradiation}

Modification of titania with only one component $(\mathrm{Ru}(\mathrm{II}), \mathrm{Au}, \mathrm{Ag})$ resulted in enhancement of photocatalytic activity during acetic acid decomposition for all of the titania samples with the exception of $\mathrm{Ru}(\mathrm{II})$-modified large rutile (STG1, $250 \mathrm{~nm}$ ), as 
shown in the left part of Fig. 11 and Table s2. The highest enhancement of photocatlytic activity (ca. 5, 8 and 9 times) was observed for large crystallites of Ag-modified titania (169, 250 and $208 \mathrm{~nm}, \mathrm{TiO}_{2}$ : Merck, STG1 and ST41, respectively). It is proposed that storage of photogenerated electrons in noble metal NPs, and thus separation of charge carriers, is the most crucial factor determining photocatalytic activity for samples with a high recombination rate of charge carriers (large titania NPs). Similarly, gold modification resulted in the highest enhancement of photocatalytic activity (ca. 6 times) for large particles of titania, i.e., STG1. A comparison of the action spectra for bare and gold-modified titania STG1 is shown in the right part of Fig. 11. Gold deposition resulted in more than a double (from ca. 3\% to 7\%) increase in the apparent quantum yield of acetic acid decomposition for irradiation in the range of 320-395 nm. Irradiation with higher energy ( $\geq 3.8 \mathrm{eV}, \lambda \leq 290 \mathrm{~nm})$ resulted in a further increase in quantum yield to $11 \%$, indicating that an additional factor, e.g., gold reshaping, was responsible for this enhancement. It has been reported that strong energy, such as UV light [40] or thermal annealing [41], resulted in gold reshaping to form more stable facets with low surface energy, i.e., spherical NPs. However, it must be pointed that this is not the case for experiments performed at irradiation with wavelengths longer than $320 \mathrm{~nm}$ (results shown in Fig. 8, left).

In the case of dual-modified titania (hybrid photocatalysts), enhancement of photocatalytic activity was observed for hybrid photocatalysts composed of Au NPs and $\mathrm{Ru}(\mathrm{II})$, while $\mathrm{Ru}(\mathrm{II})$ inhibited the photocatalytic activity of Ag-modified titania. It is thought that a second modifier could enhance the recombination of charge carriers instead of their separation as has already been reported for titania modified with core $(\mathrm{Au})$-shell $(\mathrm{Ag})$ NPs [42]. In addition, direct interaction of the ruthenium chromophore with metallic NPs could occur, and this might open an additional deactivation pathway [43]. As this binding will be affected by the nature of the metal particle, the binding group and the size of the particle, more detailed investigation is necessary.

Fig. 11. Photocatalytic activity of bare and modified titanias for acetic acid photodecomposition: left) under UV/vis irradiation $(\lambda>320 \mathrm{~nm})$; right) action spectra for bare and gold-modified titania STG1.

\subsubsection{Photocatalytic dehydrogenation of methanol under UV/vis irradiation}

Modification of titania with only one component $(\mathrm{Ru}(\mathrm{II}), \mathrm{Au}, \mathrm{Ag})$ resulted in enhancement of photocatalytic activity for all modified samples (Fig. 12 and Table s2), 
since bare titania was practically inactive for alcohol dehydrogenation. It is well known that dehydrogenation reaction occurs on the surface of co-catalysts, e.g., deposited NPs of noble metals [44]. The higher activity of gold than that of silver is directly connected to the smaller activation overpotential for evolution of hydrogen on the surface of the co-catalyst, i.e., -0.09 and $-0.022 \mathrm{~V}$, respectively. Similar results have been reported for gold-, silver- and platinum-modified titania, and it was reported that the lowest activity of silver-modified titania originated from its smallest work function, i.e. 4.74, 5.31 and $5.93 \mathrm{eV}$ for the 111 crystallite planes of $\mathrm{Ag}, \mathrm{Au}$ and $\mathrm{Pt}$, respectively $[45,46]$. The greater the difference between the metal work function and that of titania is, the higher is the electronic potential barrier (Schottky barrier) generated by the band alignment at the metal-semiconductor heterojunction, increasing the transfer and trapping of photogenerated electron by metal [47], and thus causing a higher hydrogen generation rate. The rates of hydrogen evolution also depended on the structural properties of titania and metallic NPs, and the levels of photocatalytic activity were the highest for fine anatase samples (TiO10, P25) with small gold NPs (9 and $17 \mathrm{~nm}$ ) and silver NPs (3 and $20 \mathrm{~nm}$ ), as was reported for $\mathrm{Au}-\mathrm{TiO}_{2}$ [11, 42]. It should be noted that $\mathrm{Ru}$ (II) complex also enhanced hydrogen evolution, indicating that reaction occurred on the Ru site, either by its activation under UV/vis or by accepting electrons from photo-activated titania. To clarify the mechanism, experiments are currently being carried out under single UV irradiation (in the absence of visible light).

In the case of dual-modified titania (hybrid photocatalysts), enhancement of photocatalytic activity was observed for all samples, especially those modified with $\mathrm{Ru}(\mathrm{II})$ and Ag NPs. For two gold (TIO10, Merck) and all silver hybrid photocatalysts, a small synergistic effect was noticed, indicating electronic interaction between the two modifiers. It should be pointed that for two anatase samples (ST41 and Merck), Ru(II) strongly enhanced the photocatalytic activity of almost inactive silver-modified titania, resulting in practically the same photocatalytic activity as that of gold-modified ones. This observation indicates the participation of $\mathrm{Ru}(\mathrm{II})$ in electron transfer and proton reduction.

Fig. 12. Photocatalytic activity of bare and modified titanias for methanol dehydrogenation under UV/vis irradiation $(\lambda>320 \mathrm{~nm})$.

Summarizing the photocatalytic activity under UV/vis irradiation, it must be pointed that different kinds of action were noticed for two plasmonic metals ( $\mathrm{Au}$ and $\mathrm{Ag}$ ) in two reaction systems, i.e., 
i) during oxidative decomposition of acetic acid, the $\mathrm{Ru}$ complex increased the activity of gold-modified titania and decreased the activity of silver-modified titania,

ii) during methanol dehydrogenation, the Ru complex only slightly increased the activity of gold-modified titania and greatly enhanced (synergistic effect) the activity of silver-modified titania.

There are two possible reasons for the different actions of $\mathrm{Ag}$ and $\mathrm{Au}$, i.e., photoabsorption properties or difference in surface charge on metallic NPs. LSPR of silver NPs usually appears at shorter wavelengths than that of gold NPs (for the same sizes and shapes of NPs), and thus its overlapping with MLCT of the Ru complex could result in poorer absorption properties than those of hybrid photocatalysts consisting of gold. However, it is not valid for titania P25 modified with silver possessing maximum LSPR absorption at $595 \mathrm{~nm}$, and the enhancement of activity in a methanol system on $\mathrm{Ag} / \mathrm{Ru}$ cannot be explained by poorer photoabsorption properties. Therefore, the difference in surface charges on NPs resulting in possible adsorption of the $\mathrm{Ru}$ complex on the metallic site, instead of on the titania surface, might be the reason for different activities obtained by the two metals. It is proposed that the $\mathrm{Ru}$ complex can bind to positively charged silver, since silver NPs deposited on titania are generally positively charged, in contrast to gold NPs, which are usually uncharged [42, 48]. Our XPS results confirmed that the surface of silver NPs in plasmonic and hybrid photocatalysts was positively charged “+”, while gold was mainly zero-valent.

TRMC experiments were carried out to examine the electronic character of action under UV irradiation, and the results are shown in Fig. 13. Surface modification with all modifiers strongly influenced the charge-carrier decay of titania TIO10 (Fig. 13, left). The TRMC signal is mainly related to electron mobility [28, 33, 49] since photogenerated positive holes are rapidly trapped (ns). Thus, the observed decrease in the TRMC signal is probably caused by efficient electron scavenging by both kinds of modifier, i.e., metallic NPs and Ru complex, decreasing the number of mobile electrons. All modified photocatalysts showed similar intensities of the TRMC signal, with the exception of $\mathrm{Ag} / \mathrm{Ru}$, confirming different characters of this modification, i.e., possibility of $\mathrm{Ru}(\mathrm{II})$ binding to $\mathrm{Ag} \mathrm{NPs}$, instead of to titania. To confirm this hypothesis, hybrid photocatalysts prepared in a different way, i.e., first $\mathrm{Ru}(\mathrm{II})$ binding to titania and then deposition of plasmonic metal, are presently under investigation.

The influence of TRMC signal intensity and its decay is related to photocatalytic activity, as has been reported for titania modified with noble metal NPs $[49,50]$. The good correlation (Fig. 10, right) between photocatalytic activity during 
acetic acid oxidation and the maxima of TRMC intensity $\left(\mathrm{I}_{\max }\right)$ proved that higher efficiency of electron trapping by noble metals (lower $\mathrm{I}_{\max }$ signal) resulted in higher photocatalytic activity. During methanol dehydrogenation, the correlation was not as clear because gold samples should be analyzed separately from silver ones, due to the different work functions for the two metals (as has been discussed above). For such analysis, similarly to acetic acid decomposition, enhanced scavenging of mobile electrons (lower $\mathrm{I}_{\max }$ signal) caused faster evolution of hydrogen.

Fig. 13. left) TRMC signals after excitation at $355 \mathrm{~nm}$ for bare and modified titania TIO10; right) Correlation between photocatalytic activities during acetic acid decomposition $\left(\mathrm{CO}_{2}\right.$ generation) and methanol dehydrogenation $\left(\mathrm{H}_{2}\right.$ evolution) and maximum intensity of the TRMC signal $\left(\mathrm{I}_{\max }\right)$.

\subsubsection{Photocatalytic oxidation of 2-propanol under vis irradiation $(\lambda>450 \mathrm{~nm})$}

Under visible light irradiation, fine titanias modified with $\mathrm{Ru}(\mathrm{II})$ showed the highest level of photocatalytic activity, as can be seen in Fig. 14 and in Table s2. This observation is in accordance with previously reported data for $\mathrm{Ru}(\mathrm{II})$ modification of titania with different particle sizes [33, 51]. Based on TRMC data, it was proposed that charge generation yield was less efficient for dye-sensitized films of 300-nm titania particles than for films of 20-nm titania particles [33]. The photocatalytic activity of anatase titania TIO10 modified with $\mathrm{Ag} / \mathrm{Ru}(\mathrm{II})$ exceeded the activity of the best plasmonic photocatalysts (Ag-TIO10, Au-STG1) by almost two times. Hybrid photocatalysts exhibited the same photocatalytic activities as or slightly enhanced (Ag deposited on large anatase) photocatalytic activities than those of titania modified with plasmonic NPs. However, those activities were lower than the activity of $\mathrm{Ru}$ (II)-modified titania, with the exception of $\mathrm{Ag} / \mathrm{Ru}$ (II)-modified rutile STG1, which exhibited slightly higher photocatalytic activity than that of single-modified samples (Ag-STG1 and Ru(II)-STG1). The presence of Ru(II) enhanced photocatalytic activity of almost all plasmonic photocatalysts, i.e., all silver-modified titanias and Au-ST41, Au-P25, Au-TiO10. The suggested mechanism of the action of plasmonic photocatalysts action under visible light irradiation involves charge transfer from plasmonically activated metallic NPs to the CB of titania and then to adsorbed oxygen with simultaneous oxidation of organic compounds on the surface of metallic NPs [9, 11]. Thus, the presence of an additional chromophore with the ability of electron transfer, i.e., Ru complex, resulted in photocatalytic activity enhancement under visible light irradiation. However, the reason for the decrease in photocatalytic activity for the 
most active $\mathrm{Ru}(\mathrm{II})$-modified titania after addition of plasmonic metals is unclear. It is possible that photo-exited electrons from the $\mathrm{Ru}$ complex are transferred to the $\mathrm{CB}$ of titania, from where they quickly sink in NPs of noble metals as is typically observed under UV irradiation for titania modified with noble metals (e.g., acetic acid oxidation in Fig. 11) [52]. Consequently, their transfer to adsorbed oxygen could be hindered. The function of oxygen in the reaction system based on titania modified with plasmonic NPs has not been clarified. Previously, it was found that photocatalytic activity of gold-modified titania in the absence of oxygen drastically decreased (>10x) [12]. However, it is not known whether oxygen works only as an electron acceptor or also as the source of reactive oxygen species responsible for visible light activity. Experiments in the absence of oxygen and in the presence of other electron acceptors for plasmonic, Ru-modified and hybrid photocatalysts are currently being carried out.

The hypothesis for the decrease in photocatalytic activity of hybrid photocatalysts being due to electron sinking in the NPs of noble metals could be confirmed by previously reported data on electron injection efficiency for dye-sensitized titania films. It was reported that a trap filling effect was observed in the case of a film composed of fine titania NPs $(20 \mathrm{~nm})$ [33], i.e., increase of electron mobility due to a trapping/detrapping mechanism by shallow traps. However, for large titania particles of $300 \mathrm{~nm}$, no trap filling effect was observed. It is proposed that electrons are irreversible captured by deep traps in the case of large titania particles. NPs of a noble metal could hinder this trapping process by fast electron scavenging that resulted in enhancement of photocatalytic activity of hybrid photocatalysts composed of large titania STG1. However, in the case of fine titania possessing a large amount of shallow traps (high electron mobility), the presence of noble metal NPs may hinder the traping/detrapping mechanism. Consequently, noble metal NPs reduced electron mobility, which was confirmed by TRMC data (after UV excitation). A similar mechanism of electron transfer, from the sensitizer via titania to the metal, was also proposed for Co-modified titania sensitized with Rhodamine B [53]. It was observed that co-existence of metal NPs and an organic sensitizer facilitated water reduction. It must be pointed that water reduction occurs on the surface of metal NPs (the same as methanol dehydrogenation discussed in section 3.2.2), and therefore enhancement of photocatalytic activity is expected for all hybrid photocatalysts (study now in progress).

Fig. 14. Photocatalytic activities of bare and modified titanias for 2-propanol oxidation under vis irradiation $(\lambda>450 \mathrm{~nm})$. 


\section{Conclusions}

The anchoring groups of $\mathrm{Ru}(\mathrm{II})$ complexes and structural properties of titania are crucial for efficient complex adsorption. The degree of deprotection of phosphonate groups and the use of titania with fine crystallites and large surface area are important for efficient $\mathrm{Ru}$ (II) binding. On the other hand, the titania crystalline form (anatase or rutile) does not play an important role.

Modification of titania with either NPs of noble metals or $\mathrm{Ru}(\mathrm{II})$ complexes causes photocatalytic activity enhancement under UV/vis irradiation, due to separation of charge carriers, as was proved by TRMC experiments. Enhancement or inhibition of photocatalytic activity after dual modification (for hybrid photocatalysts) is strongly dependent on the kind of noble metal. It is proposed that, due to different surface charges on metallic NPs of gold and silver, i.e., silver NPs are mainly positively charged, while gold NPs are zero-valent, Ru(II) can bind also to the surface of silver NPs.

An electronic interaction between two modifiers has been proposed since a synergistic effect was observed for hybrid photocatalysts composed of $\mathrm{Ru}(\mathrm{II})$ and silver NPs during photocatalytic dehydrogenation of methanol.

Under visible light irradiation, the opposite tendency for two modifiers was observed, i.e., enhancement of photocatalytic activity of metal-modified titania by the $\mathrm{Ru}$ complex and decrease of high photocatalytic activity of $\mathrm{Ru}(\mathrm{II})$ by co-deposited NPs of a noble metal. The reason for the contradictory actions of the two modifiers is unclear and will be further examined, e.g., by action spectrum analysis. Preparation of hybrid photocatalysts in which adsorption of $\mathrm{Ru}(\mathrm{II})$ on metallic sites will be avoided, i.e., first $\mathrm{Ru}$ (II) will be adsorbed on titania and then plasmonic metal will be deposited, is also presently being investigated.

The results for various photocatalytic reactions highlight the fact that separate optimization of hybrid catalysts is necessary to generate highly active photocatalysts utilizing the full potential of the synergistic effects of independent light harvesters and catalytic centers. The interplay between charge carrier mobility, nature of titania, nature of NPs and type of ruthenium complex is highly complex. Combined investigation is essential for unraveling the chemical and photochemical processes determining the activity.

\section{Acknowledgements}

Financial support from Bill \& Melinda Gates Foundation through the Grand Challenges Exploration Initiative (OPP1060234) and the CONCERT-Japan Joint Call on Efficient 
Energy Storage and Distribution/Resilience against Disasters is highly acknowledged. The authors are grateful to Prof. Christophe Colbeau-Justin, Mr. Baris Karabiyik, Ms. Christina Muedsan and Mr. Jonathan Verret for technical assistance and discussion on TRMC analysis.

\section{References}

[1] M.R. Hoffmann, S.T. Martin, W.Y. Choi, D.W. Bahnemann, Chem. Rev. 95 (1995) 69-96.

[2] B. Ohtani, Chem. Lett. 37 (2008) 217-229.

[3] J. Grzechulska, M. Hamerski, A.W. Morawski, Water Res. 34 (2000) 1638-1644.

[4] A.J. Bard, J. Photochem. 10 (1979) 59-75.

[5] A. Dawson, P.V. Kamat, J. Phys. Chem. B 105 (2001) 960-966.

[6] B. Ohtani, M. Kakimoto, S. Nishimoto, T. Kagiya, J. Phys. Chem. A: Chem. 70 (1993) 265-272.

[7] M.V. Dozzi, L. Prati, P. Canton, E. Selli, Phys. Chem. Chem. Phys. 11 (2009) 7171-7180.

[8] W. Macyk, H. Kisch, Chem. - Eur. J. 7 (2001) 1862-1867.

[9] Y. Tian, T. Tatsuma, J. Am. Chem. Soc. 127 (2005) 7632-7637.

[10] M. Haruta, Catal. Today. 36 (1997) 153-166.

[11] E. Kowalska, R. Abe, B. Ohtani, Chem. Commun. (2009) 241-243.

[12] E. Kowalska, O.O.P. Mahaney, R. Abe, B. Ohtani, Phys. Chem. Chem. Phys. 12 (2010) 2344-2355.

[13] E. Kowalska, S. Rau, B. Ohtani, Journal of Nanotechnology 2012 (2012) 1-11.

[14] C.J. Orendorff, T.K. Sau, C.J. Murphy, Small 2 (2006) 636-639.

[15] H. Wang, D.W. Brandl, F. Le, P. Nordlander, N.J. Halas, Nano. Lett. 6 (2006) 827-832.

[16] Y.N. Xia, Y.J. Xiong, B. Lim, S.E. Skrabalak, Angew. Chem. Int. Edit. 48 (2009) 60-103.

[17] B. O'Regan, M. Graetzel, Nature 353 (1991) 737-740.

[18] P. Wang, S.M. Zakeeruddin, J.E. Moser, M.K. Nazeeruddin, T. Sekiguchi, M. Graetzel, Nat. Mater. 2 (2003) 402-407.

[19] B. O'Regan, F. Lenzmann, R. Muis, J. Wienke, Chem. Mater. 14 (2002) 5023-5029.

[20] S. Rau, D. Walther, J.G. Vos, Dalton Trans. （2007) 915-919.

[21] K. Halbauer, A. Gobel, A. Sterzik, H. Gorls, S. Rau, W. Imhof, Eur. J. Inorg. Chem. (2007) 1508-1514.

[22] E. Bae, W. Choi, J. Park, H.S. Shin, S.B. Kim, J.S. Lee, J. Phys. Chem. B 108 (2004) 
14093-14101.

[23] S.A. Trammell, J.A. Moss, J.C. Yang, B.M. Nakhle, C.A. Slate, F. Odobel, M. Sykora, B.W. Erickson, T.J. Meyer, Inorg. Chem. 38 (1999) 3665-3669.

[24] G. Sprintschnik, H.W. Sprintschnik, P.P. Kirsch, D.G. Whitten, J. Am. Chem. Soc. 99 (1977) 4947-4954.

[25] I. Gillaizeau-Gauthier, F. Odobel, M. Alebbi, R. Argazzi, E. Costa, C.A. Bignozzi, P. Qu, G.J. Meyer, Inorg. Chem. 40 (2001) 6073-6036.

[26] H. Zabri, I. Gillaizeau, C.A. Bignozzi, S. Caramori, M.F. Charlot, J. Cano-Boquera, F. Odobel, Inorg. Chem. 42 (2003) 6655-6666.

[27] L.A. Gallagher, S.A. Serron, X.G. Wen, B.J. Hornstein, D.M. Dattelbaum, J.R. Schoonover, T.J. Meyer, Inorg. Chem. 44 (2005) 2089-2097.

[28] C. Colbeau-Justin, M. Kunst, D. Huguenin, J. Mater. Sci. 38 (2003) 2429.

[29] F. Izumi, K. Momma, Solid State Phenom. 130 (2007) 15.

[30] E. Kowalska, S. Rau, Recent Patents on Engineering 4 (2010) 242-266.

[31] E. Bae, W. Choi, J. Phys. Chem. B 110 (2006) 14792-14799.

[32] Y.M. Cho, W.Y. Choi, C.H. Lee, T. Hyeon, H.I. Lee, Environ. Sci. Technol. 35 (2001) 966-970.

[33] R. Katoh, A. Huijser, K. Hara, T.J. Savenije, L.D.A. Siebbeles, J. Phys. Chem. C 111 (2007) 10741-10746.

[34] L. Li, L.L. Duan, Y.H. Xu, M. FGorlov, A. Hagfeldt, L.C. Sun, Chem. Commun. (2010) 7307-7309.

[35] E. Kowalska, L. Rosa, S. Rau, S. Juodkazis, B. Ohtani, Chem. Commun. (in preparation).

[36] B.K. Min, W.T. Wallance, D.W. Goodman, Surf. Sci. 600 (2006) L7.

[37] B. Ohtani, O.O. Prieto-Mahaney, D. Li, R. Abe, J. Photoch. Photobiol. 216 (2010) 179-182.

[38] N. Murakami, R. Abe, B. Ohtani, Chem. Phys. Lett. 451 (2008) 316-320.

[39] O.O. Prieto-Mahaney, N. Murakami, R. Abe, B. Ohtani, Chem. Lett. 38 (2009) 238-239.

[40] E.-S.A.M. Al-Sherbini, Mater. Chem. Phys. 121 (2010) 349-353.

[41] Y. Liu, E.N. Mills, R.J. Composto, J. Mater. Chem. 19 (2009) 2704-2709.

[42] E. Kowalska, M. Janczarek, L. Rosa, S. Juodkazi, B. Ohtani, Catal. Today 230 (2014) 131-137.

[43] L. Zedler, F. Theil, A. Csaki, W. Fritzsche, S. Rau, M. Schmitt, J. Popp, B. Dietzek, RSC Adv. 2 (2012) 4463-4471.

[44] B. Ohtani, K. Iwai, S.-i. Nishimoto, S. Sato, J. Phys. Chem. B 101 (1997) 
3349-3359.

[45] G.L. Chiarello, M.H. Aguirre, E. Selli, J. Catal. 273 (2010) 182-190.

[46] A. Sclafani, M.N. Mozzanega, P. Pichat, J. Photochem. Photobiol. A: Chem. 59 (1991) 181-189.

[47] A.L. Linsebigler, G. Lu, J.T. Yates, Jr., Chem. Rev. 95 (1995) 735-758.

[48] A. Zielińska-Jurek, E. Kowalska, J.W. Sobczak, W. Lisowski, B. Ohtani, A. Zaleska, Appl. Catal. B-Environ. 101 (2011) 504-514.

[49] Z.B. Hai, N. El Kolli, D.B. Uribe, P. Beaunier, M. Jose-Yacaman, J. Vigneron, A. Etcheberry, S. Sorgues, C. Colbeau-Justin, J.F. Chena, H. Remita, J. Mater. Chem. A 1 (2013) 10829-10835.

[50] E. Kowalska, H. Remita, C. Colbeau-Justin, J. Hupka, J. Belloni, J. Phys. Chem. C 112 (2008) 1124-1131.

[51] Y. Tachibana, K. Hara, S. Takano, K. Sayama, H. Arakawa, Chem. Phys. Lett. 364 (2002) 297-302.

[52] V. Subramanian, E. Wolf, P.V. Kamat, J. Phys. Chem. B 105 (2001) 11439-11446.

[53] T.T. Le, M.S. Akhtar, D.M. Park, J.C. Lee, O.B. Yang, Appl. Catal. B-Environ. 111 (2012) 397-401. 


\section{LIST OF TABLES}

\section{Table 1}

Details of experimental equipment and operation parameters used for sample characterization

Table 2

Properties of hybrid photocatalysts

\section{LIST OF FIGURES}

Fig. 1. $\mathrm{Ru}(\mathrm{II}) \mathrm{Carb}$ adsorption on titania P25 in acetonitrile $(0.16 \mathrm{~mol} \%)$ : left $) \mathrm{UV} / \mathrm{vis}$ absorbance spectra of the supernatant for $0,1.5,3.5,7,23,25,28$ and $60 \mathrm{~h}$ of adsorption; right) Adsorption efficiency; inset) Chemical structure of $\mathrm{Ru}(\mathrm{II}) \mathrm{Carb}$.

Fig 2. Adsorption efficiency of Ru(II)Phos on anatase (ST01, closed symbols) and rutile (Ald_R), open

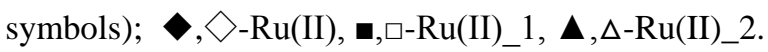

Fig. 3. Representative UV/vis absorbance spectra of the supernatant during Ru(II) adsorption on anatase titania: ST01 (left) and ST41 (right).

Fig. 4. left) Effects of crystallite size $(\boldsymbol{\Delta}, \Delta)$ and specific surface area $(\bullet, \circ)$ of titania on efficiency of $\mathrm{Ru}(\mathrm{II})$ adsorption after $2 \mathrm{~h}$ of mixing: anatase (closed symbols) and rutile (open symbols) titania; inset: STEM image of bare rutile (Ald_R); right) STEM image of bare anatase (ST01).

Fig. 5. Influence of pH on adsorption efficiency of Ru(II) on titania ST01 (left) and Ald_R (right).

Fig. 6. Influence of pre-deposited metals on $\mathrm{Ru}(\mathrm{II})$ binding efficiency during long (left) and short (right) time adsorption.

Fig. 7. XRD patterns of bare, silver-modified and gold-modified titania: top) rutile STG1, bottom) anatase ST41; insets) enlargement showing (111) and (200) facets of gold and silver.

Fig. 8. Absorption spectra (DRS) of bare and modified titania: (a) STG1, (b) P25, (c) Merck, d) ST41 and (e) TIO10.

Fig. 9. STEM images of silver-modified (left) and gold-modified (right) titania STG1 (top) and ST41 (bottom).

Fig. 10. XPS data of titania P25 modified with silver, gold and Ru(II); representative XPS spectra for P25 modified with A) Ag/Ru(II) and B) Au.

Fig. 11. Photocatalytic activity of bare and modified titanias for acetic acid photodecomposition: left) under $U V /$ vis irradiation $(\lambda>320 \mathrm{~nm})$; right) action spectra for bare and gold-modified titania STG1.

Fig. 12. Photocatalytic activity of bare and modified titanias for methanol dehydrogenation under UV/vis irradiation $(\lambda>320 \mathrm{~nm})$.

Fig. 13. left) TRMC signals after excitation at $355 \mathrm{~nm}$ for bare and modified titania TIO10; right) Correlation between photocatalytic activities during acetic acid decomposition $\left(\mathrm{CO}_{2}\right.$ generation) and methanol dehydrogenation $\left(\mathrm{H}_{2}\right.$ evolution) and maximum intensity of the TRMC signal $\left(\mathrm{I}_{\max }\right)$. 
Fig. 14. Photocatalytic activities of bare and modified titanias for 2-propanol oxidation under vis irradiation $(\lambda>450 \mathrm{~nm})$. 

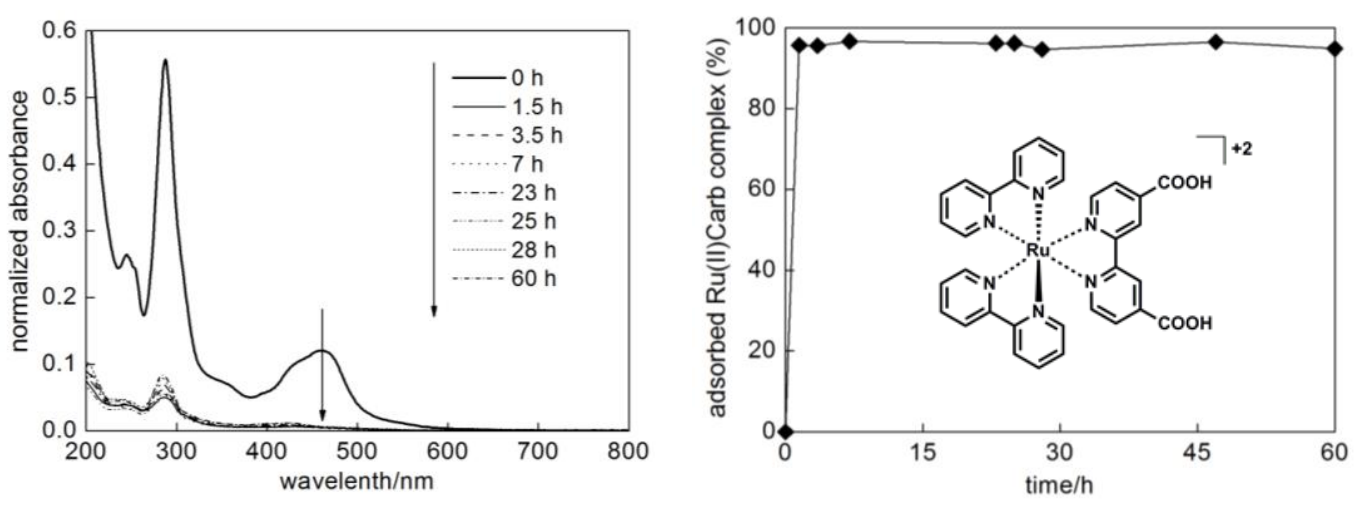

Fig. 1 


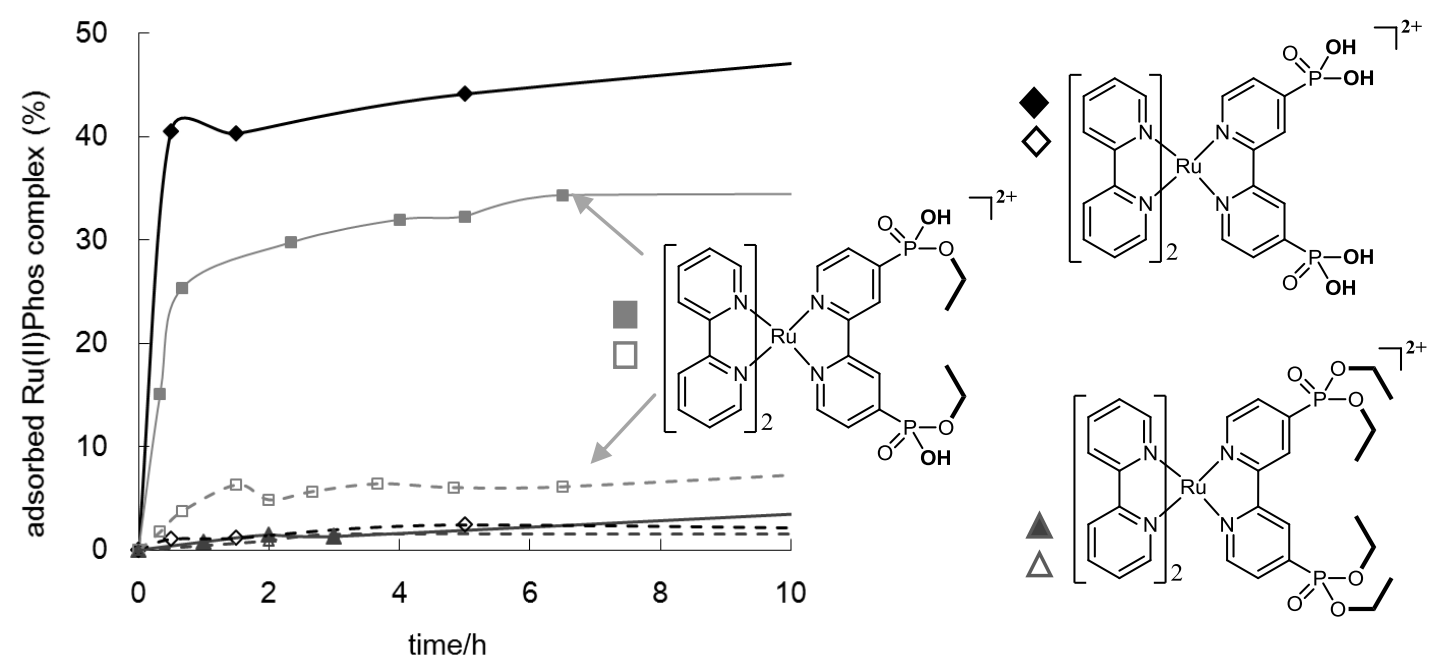

Fig. 2 

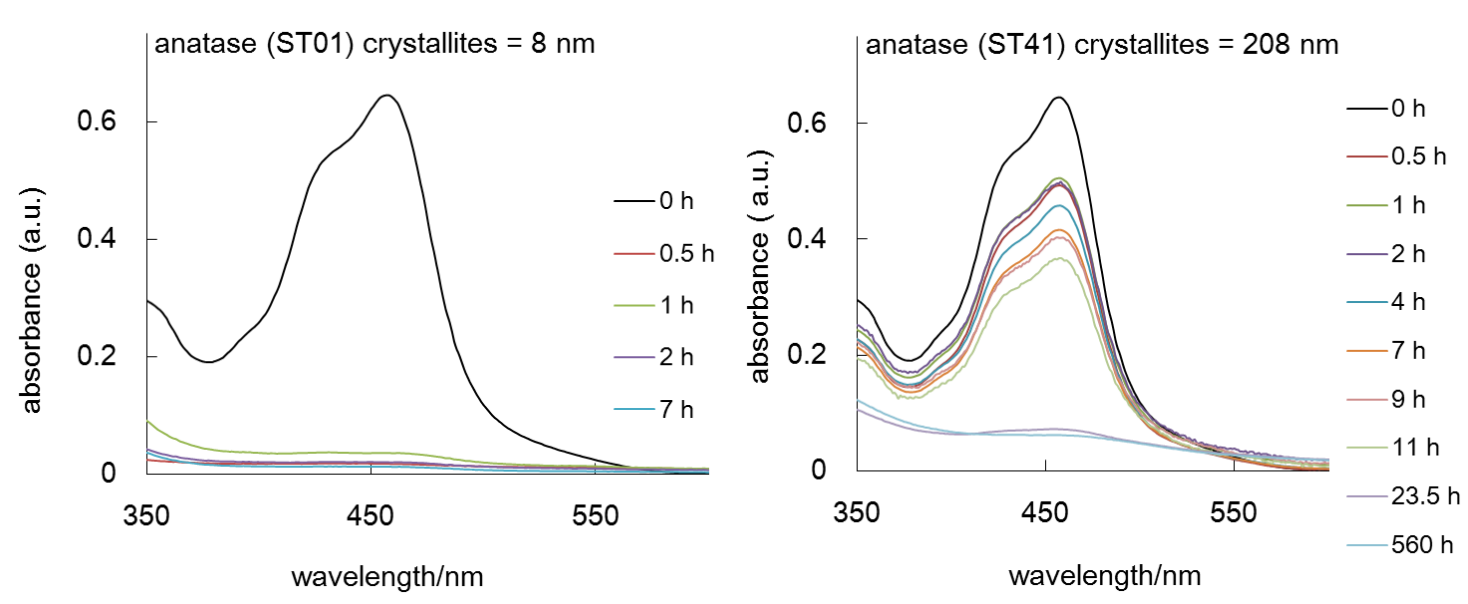

Fig. 3 


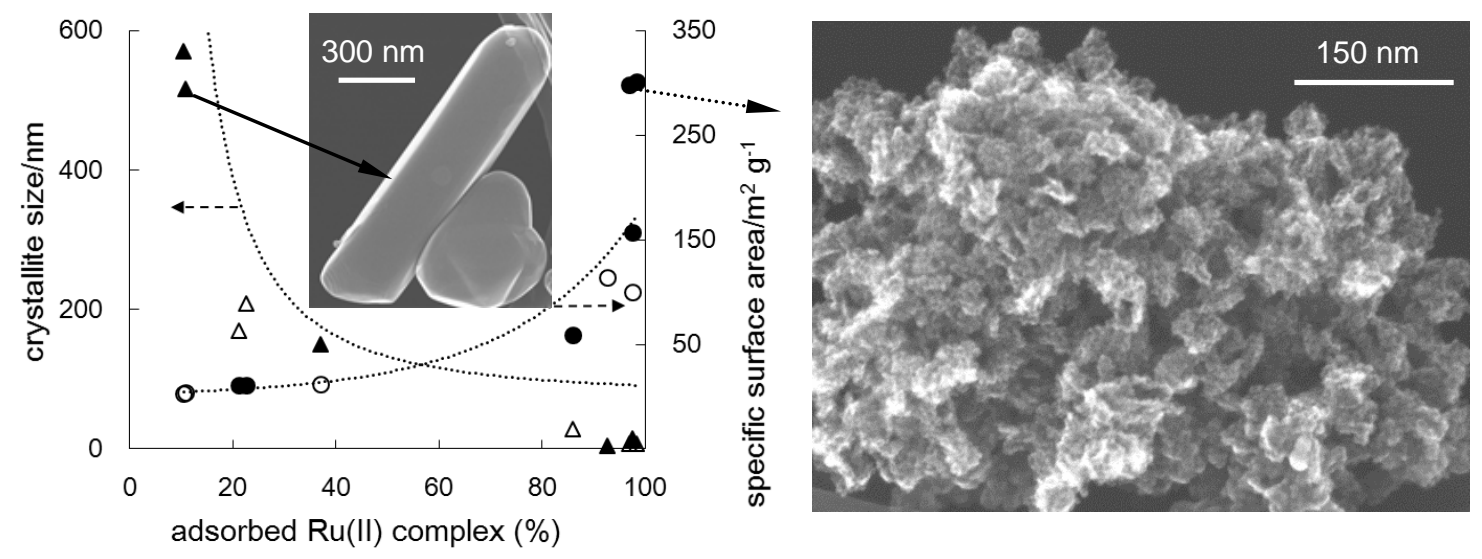

Fig. 4 

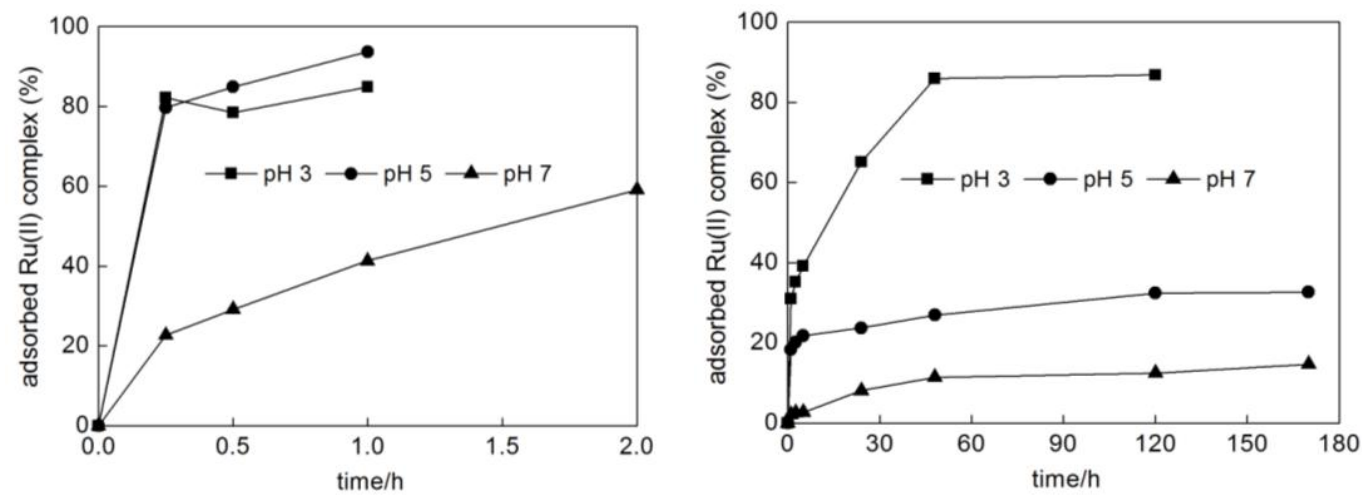

Fig. 5 

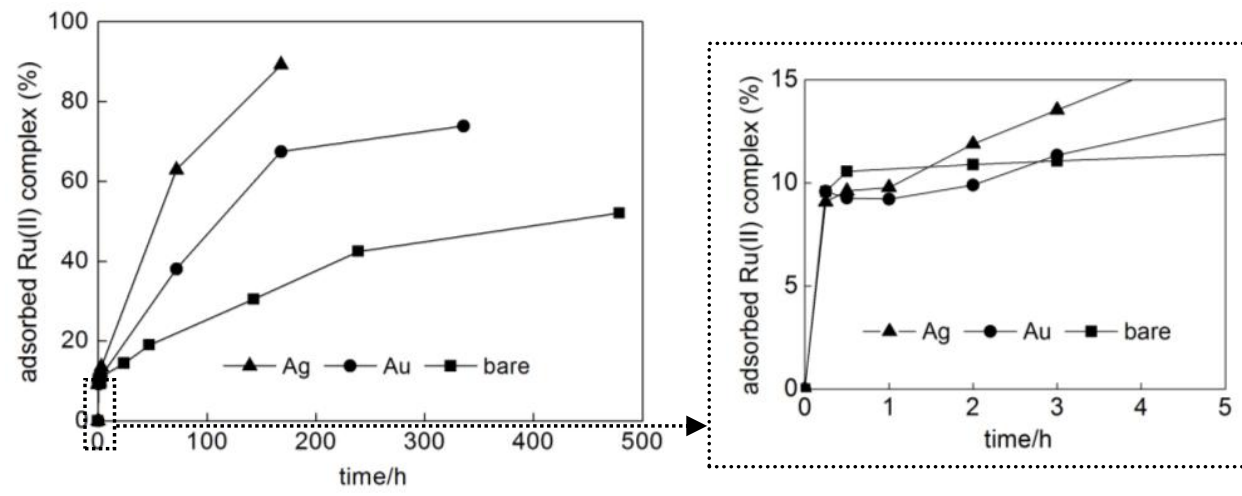

Fig. 6 

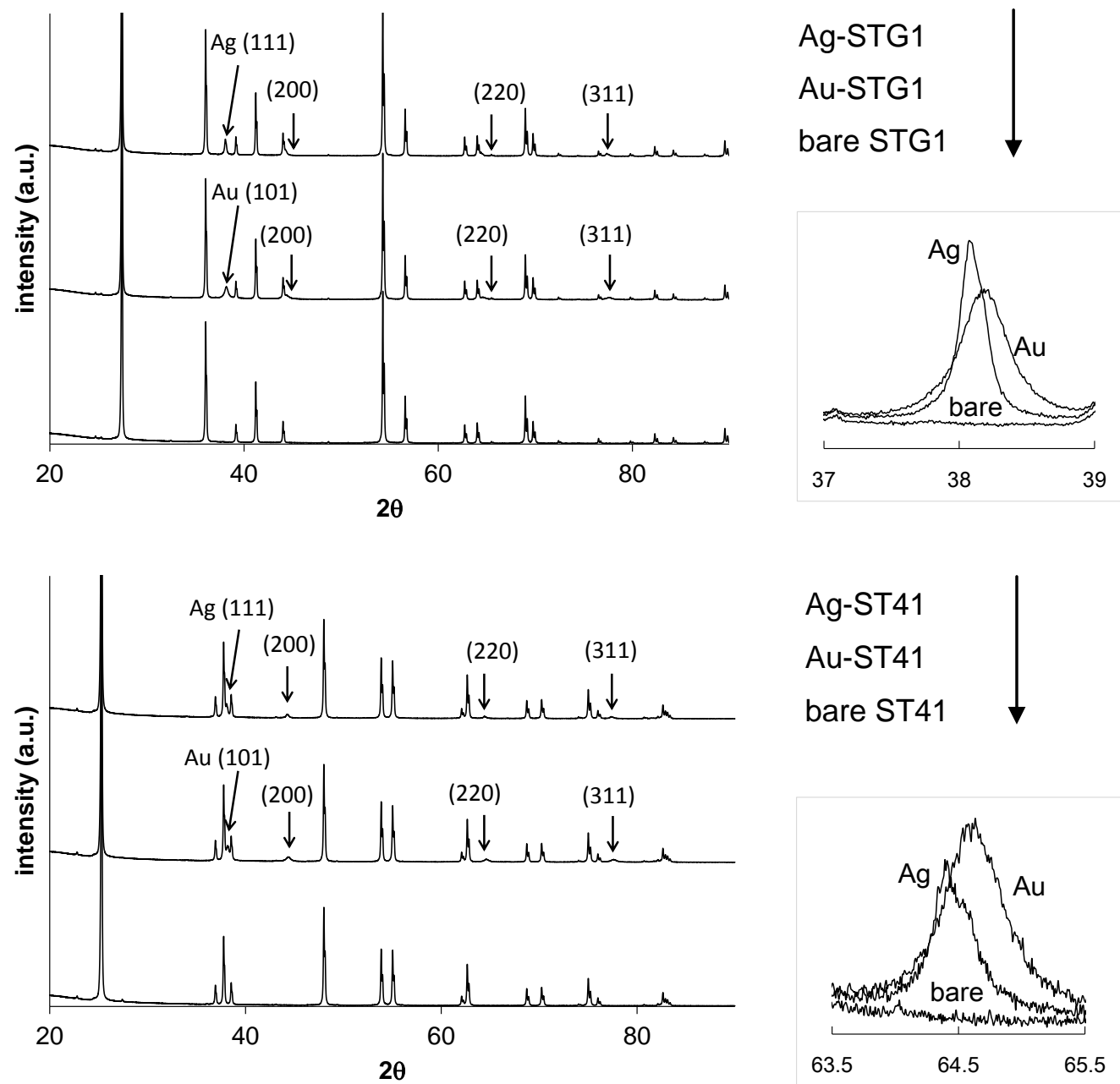

Fig. 7 
(a)

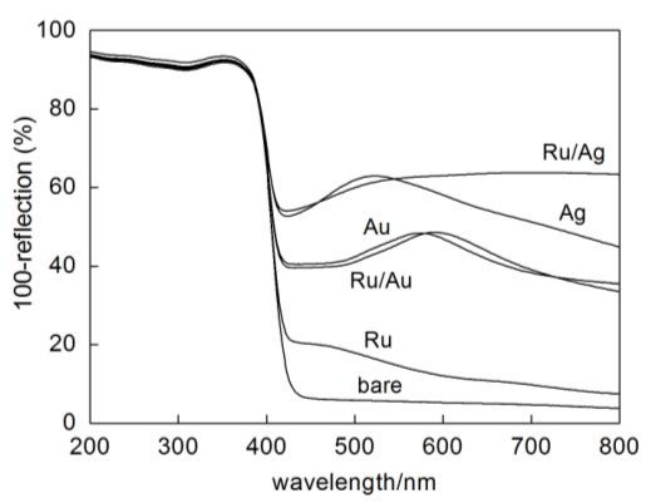

(c)

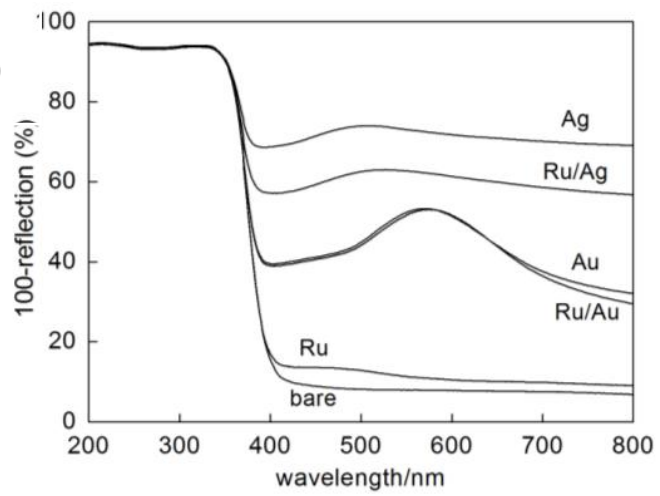

(e)

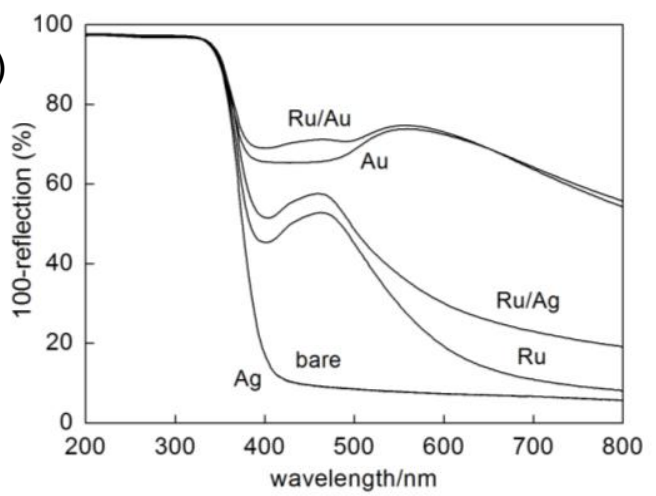

Fig. 8 (b)

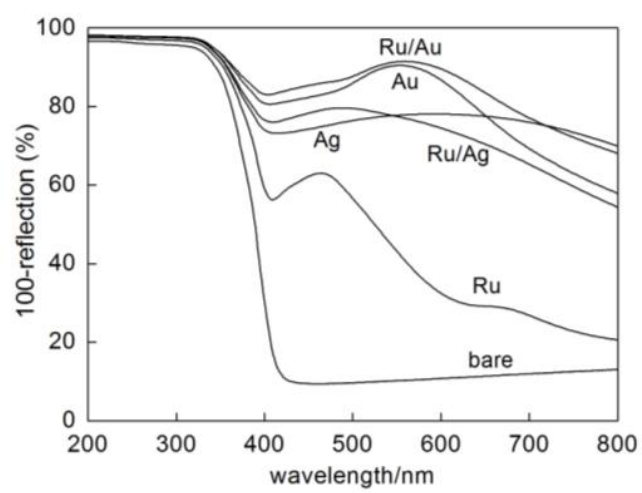

(d)

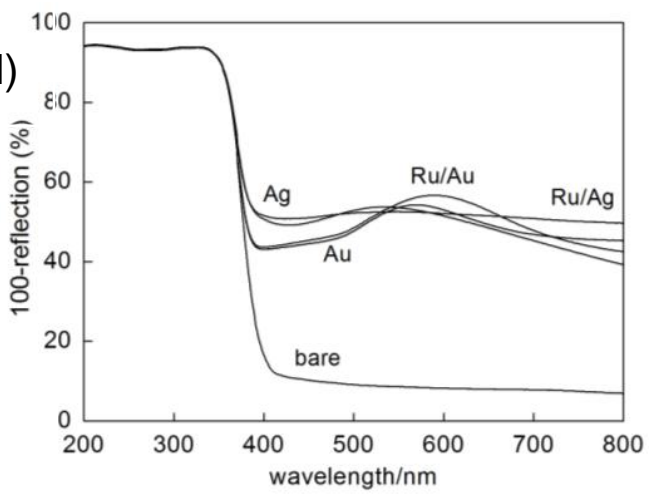

30 


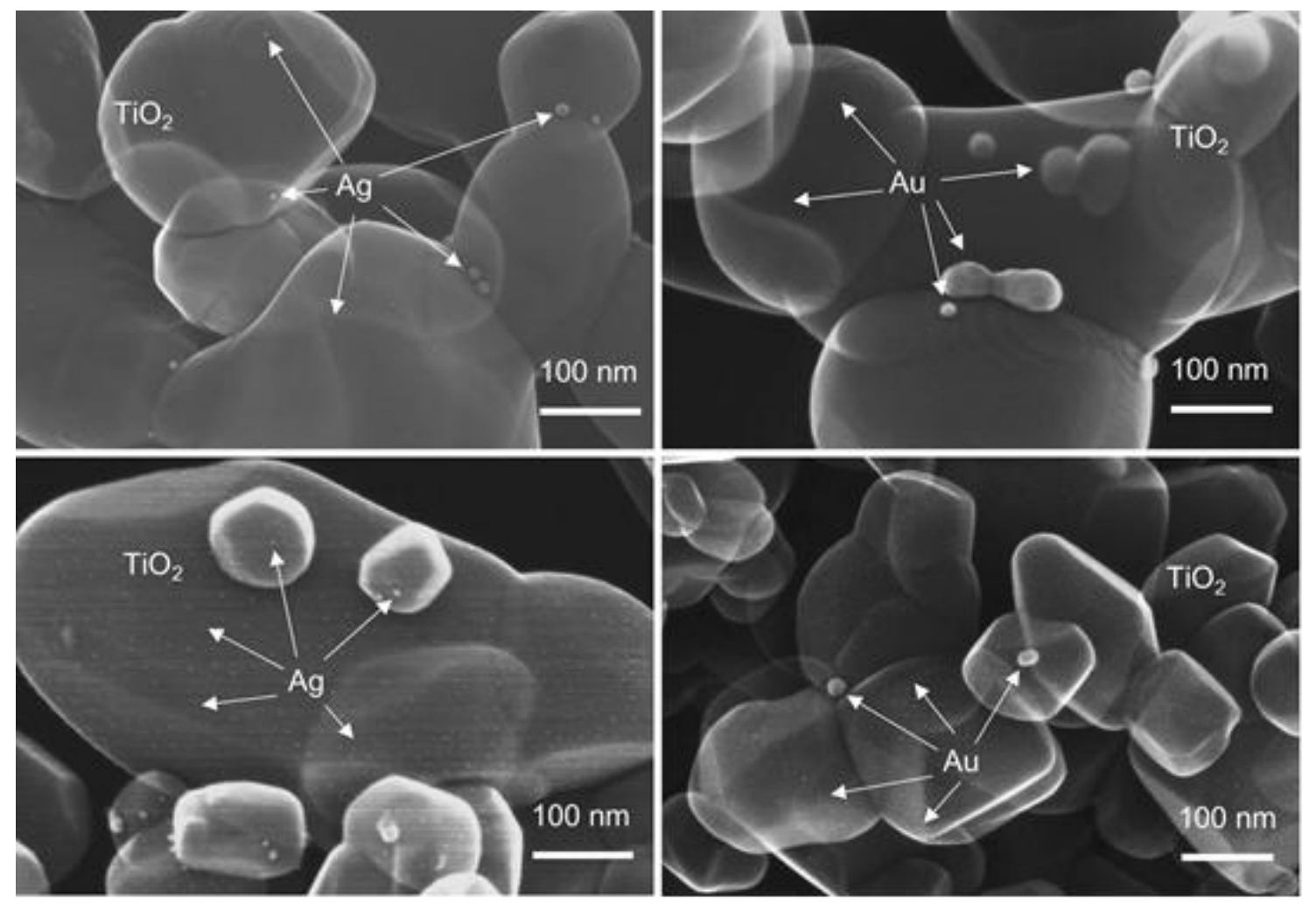

Fig. 9 


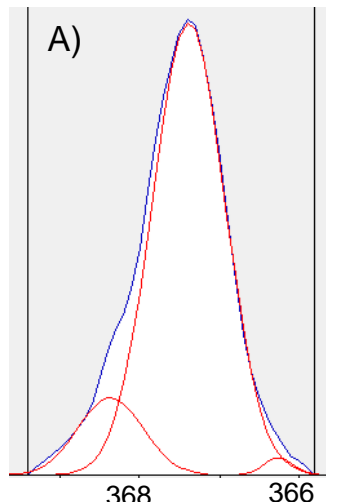

$\mathrm{Ag} 3 \mathrm{~d} 5 / 2 \mathrm{BE}(\mathrm{eV})$

366

\begin{tabular}{|c|c|c|c|}
\hline Sample & \multicolumn{3}{|c|}{ Ag content (at.\%) } \\
\hline & $\mathrm{Ag}^{0}(368.4 \mathrm{eV})$ & $\mathrm{Ag}^{+}(367.4 \mathrm{eV})$ & $\mathrm{Ag}^{+2}(366.3 \mathrm{eV})$ \\
\hline $\mathrm{Ag}$ & 11.44 & 88.29 & 0.27 \\
\hline \multirow[t]{4}{*}{$\mathrm{Ag} / \mathrm{Ru}(\mathrm{II})$} & 13.27 & 85.45 & 1.28 \\
\hline & \multicolumn{3}{|c|}{ Au content (at.\%) } \\
\hline & $\mathrm{Au}^{\delta+}(83.6)$ & $\mathrm{Au}^{0}(82.8)$ & $\mathrm{Au}^{\delta-}(81.8)$ \\
\hline & 83.6 & 82.8 & 81.8 \\
\hline $\mathrm{Au}$ & 1.68 & 89.39 & 8.93 \\
\hline $\mathrm{Au} / \mathrm{Ru}(\mathrm{II})$ & 2.58 & 97.42 & - \\
\hline
\end{tabular}

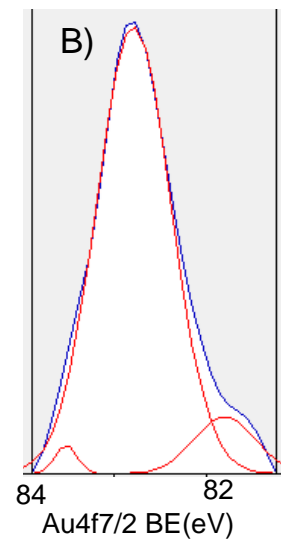

Fig. 10 

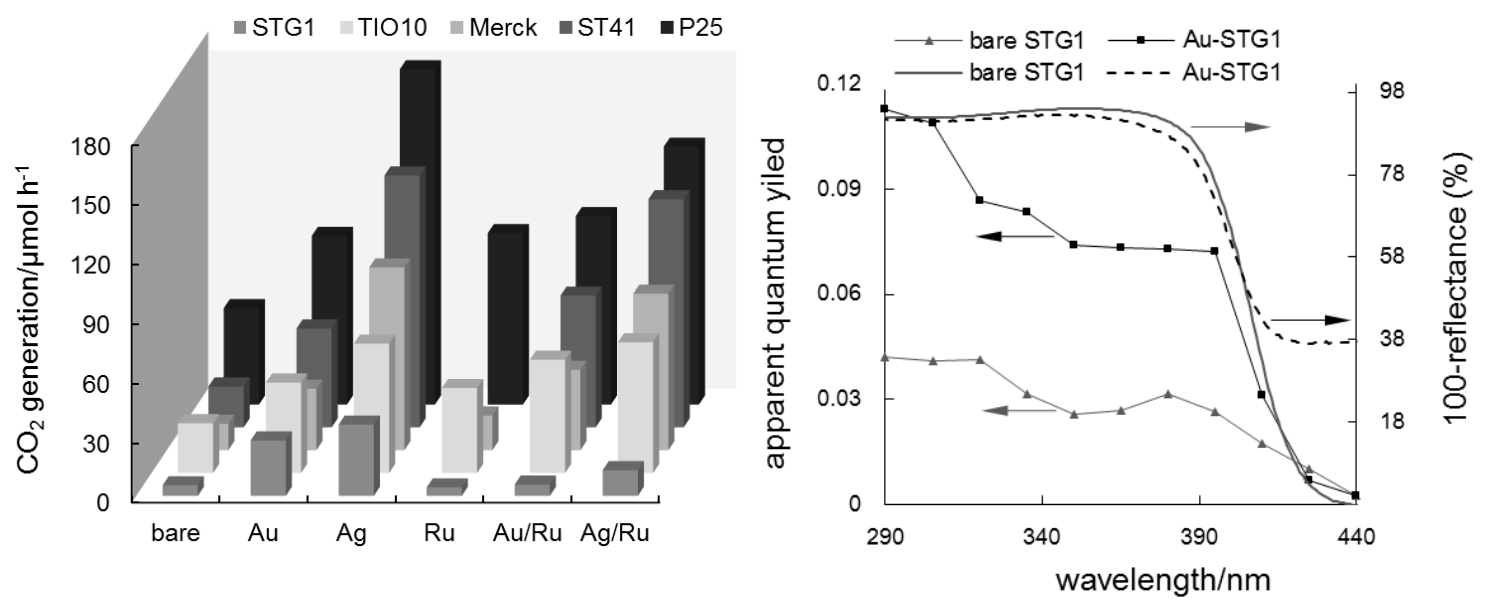

Fig. 11 


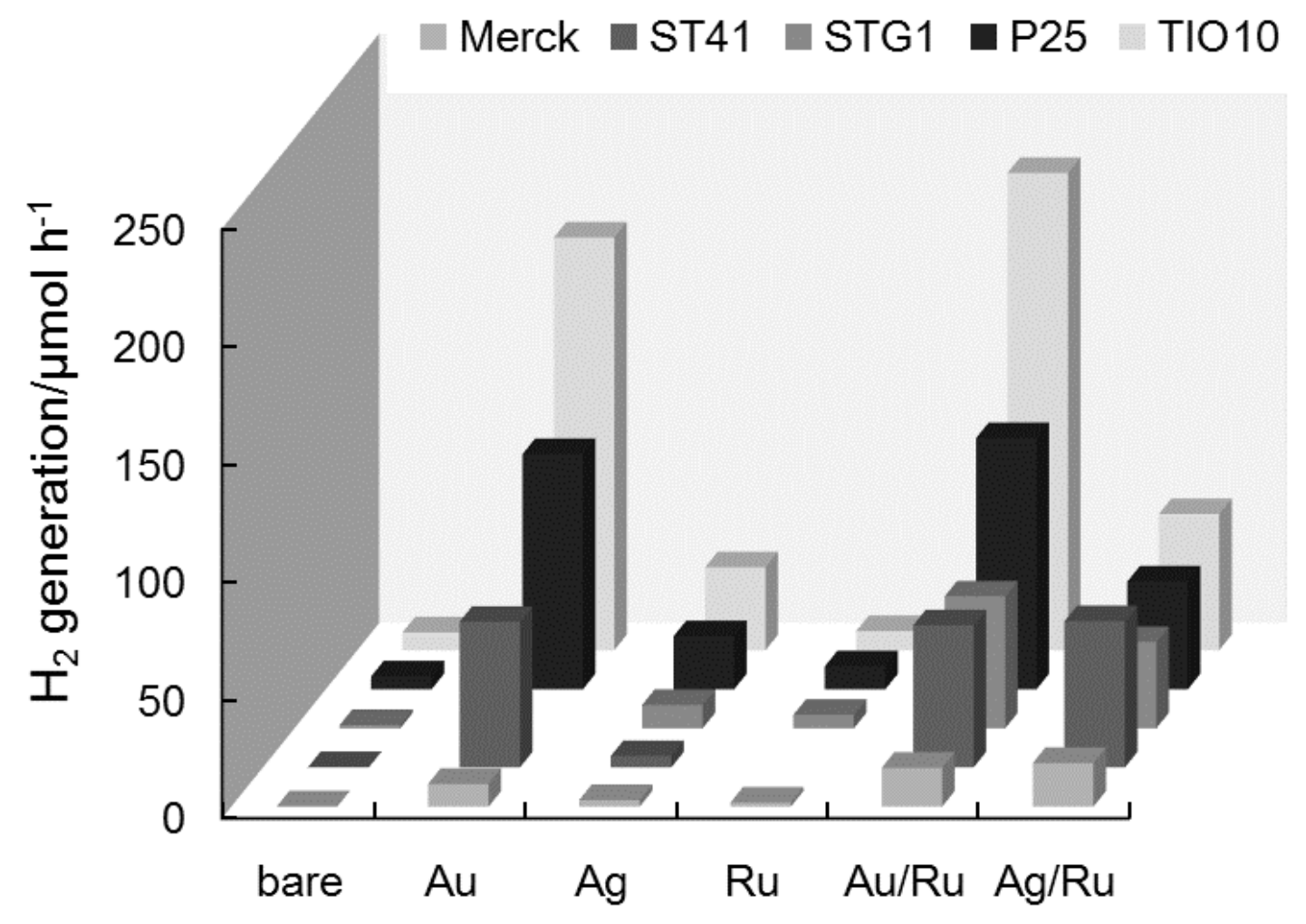

Fig. 12 

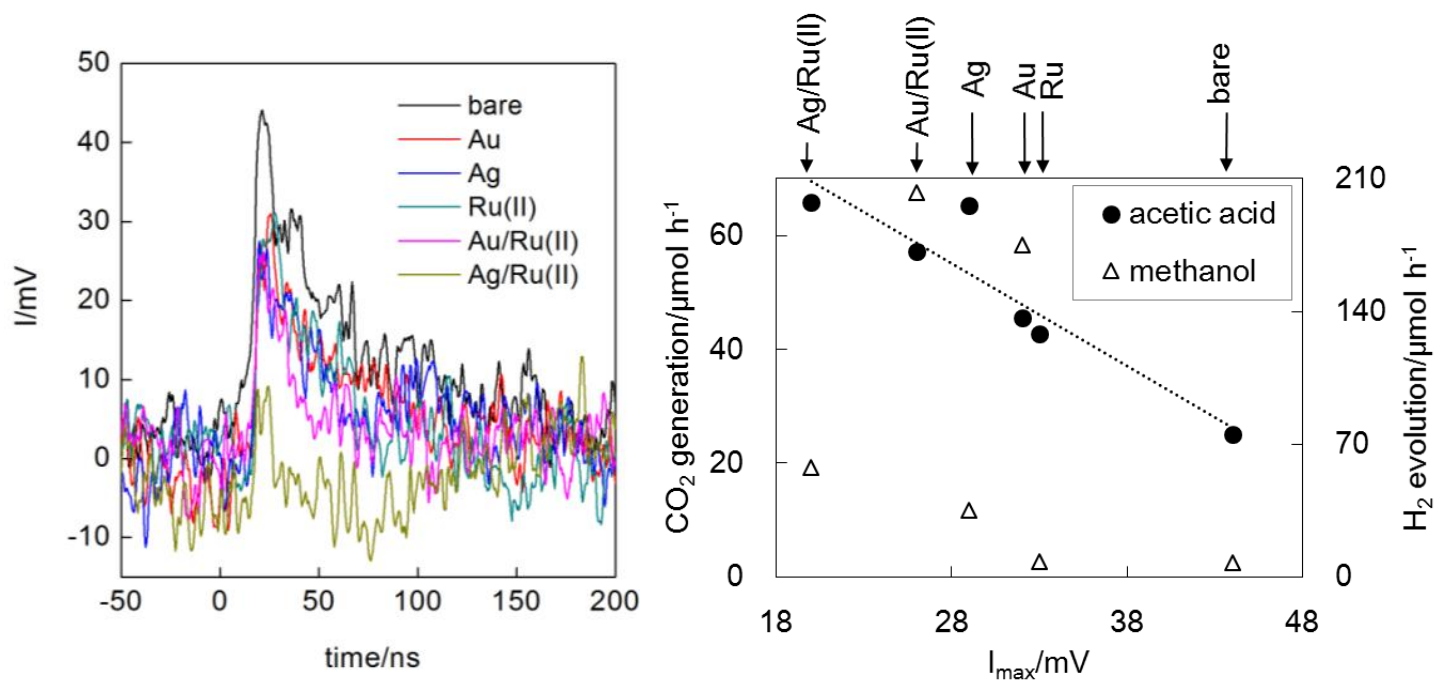

Fig. 13 


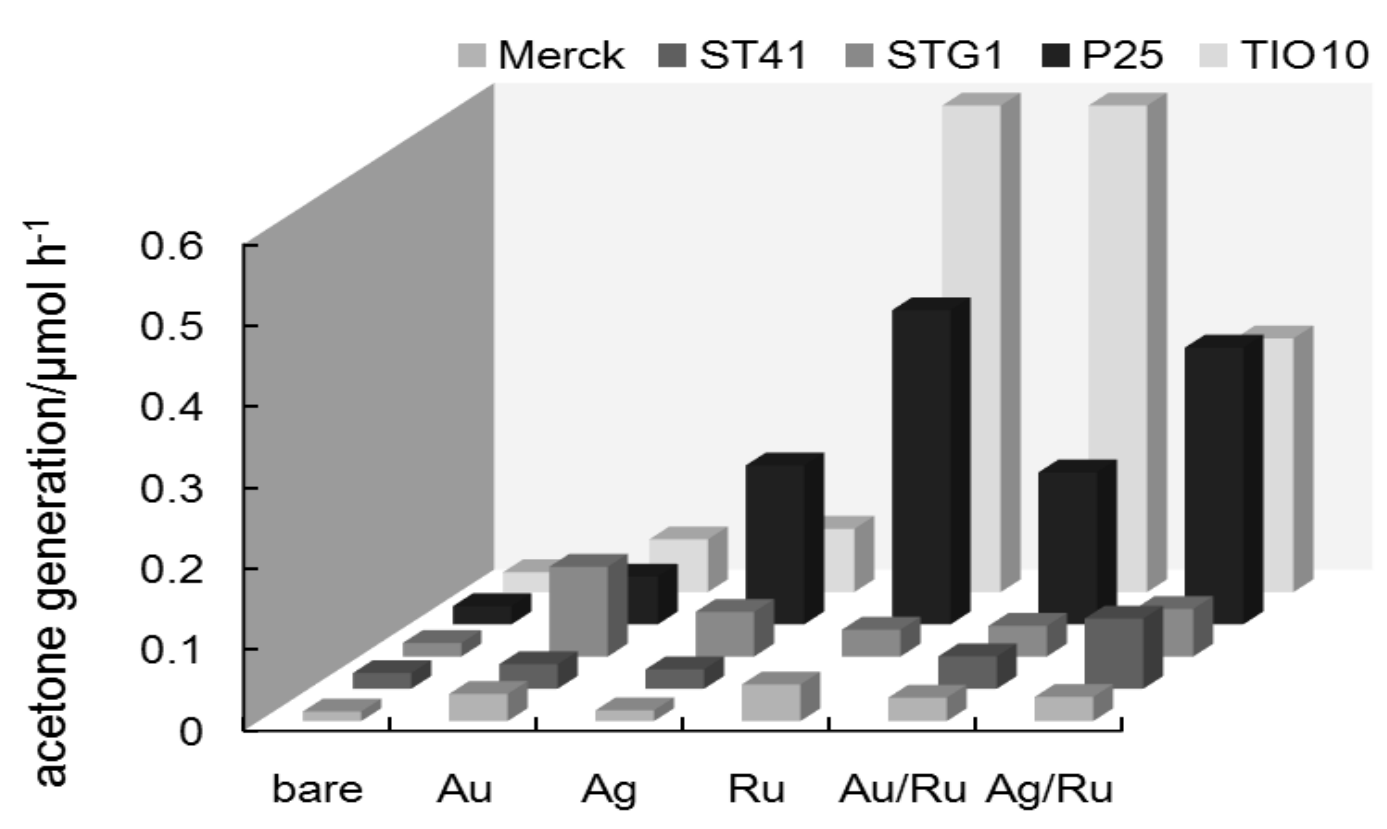

Fig. 14 


\section{Table 1.}

\begin{tabular}{|c|c|}
\hline Method & Equipment type and operation parameters \\
\hline $\mathrm{UV} / \mathrm{vis}$ & Varian Cary 50; 200-800 nm \\
\hline BET & Yuasa-Quantachrome NOVA 1200e surface and pore analyzer \\
\hline DRS & $\begin{array}{l}\text { JASCO V-670 equipped with a PIN-757 integrating sphere; Poly(tetrafluoroethylene) } \\
\text { reference, barium sulfate and bare titania used as reference }\end{array}$ \\
\hline $\mathrm{XRD}$ & $\begin{array}{l}\text { Rigaku Intelligent XRD system, SmartLab equipped with a sealed tube X-ray generator } \\
\text { (a copper target, } 40 \mathrm{kV}, 30 \mathrm{~mA} \text { ), a D/teX high-speed position sensitive detector system } \\
\text { and an ASC-10 automatic sample changer; } 2 \theta \text { range: } 10-90^{\circ} \text {, scan speed: } 1.00^{\circ} \mathrm{min}^{-1} \text {, } \\
\text { scan step } 0.008^{\circ} \text {; XRD patterns analyzed by Rigaku PDXL; A crystal structure analysis } \\
\text { package including Rietveld analysis (RIETAN FP [29]) }\end{array}$ \\
\hline STEM & $\begin{array}{l}\text { HITACHI HD2000 ultrathin evaluation system ( } 200 \mathrm{kV} \text { accelerating voltage, } 30 \mu \mathrm{A} \\
\text { emission current); Powders were dispersed in ethanol in an ultrasonic bath for a few } \\
\text { minutes and suspension droplets were deposited on a carbon covered copper microgrid } \\
\text { (Onken, types A and B); Images were acquired at wide range of magnification } \\
(200,000-800,000) \text { in normal resolution modes as secondary electron (SE), Z-contrast } \\
(\mathrm{ZC}) \text {, and bright-field (BF) modes }\end{array}$ \\
\hline XPS & JEOL JPC-9010MC, MgK $\alpha$ X-ray \\
\hline${ }^{1} \mathrm{H}-\mathrm{NMR}$ & JEOL $270 \mathrm{MHz} ; 270 \mathrm{MHz}$ in $\mathrm{CD}_{3} \mathrm{CN}, \mathrm{MeOD}$ and $\mathrm{D}_{2} \mathrm{O}$ \\
\hline 2D-COSY & JEOL $400 \mathrm{MHz} ; 400 \mathrm{MHz}$ in $\mathrm{MeOD}$ and $\mathrm{D}_{2} \mathrm{O}$ \\
\hline
\end{tabular}


Table 2

\begin{tabular}{llllllllll}
\hline & \multicolumn{1}{c}{ BET } & \multicolumn{3}{c}{ Crystalline size/nm } & \multicolumn{2}{c}{ LSPR/nm } & \multicolumn{3}{c}{$\mathrm{TiO}_{2}$ composition (\%) } \\
& $/ \mathrm{m}^{2} \mathrm{~g}^{-1}$ & $\mathrm{TiO}_{2}$ & $\mathrm{Au}$ & $\mathrm{Ag}$ & $\mathrm{Au}$ & $\mathrm{Ag}$ & anatase & rutile & non-crystal \\
\hline ST41 & 10.8 & $208^{*}$ & 29.2 & 33.8 & 570 & 532 & 98.2 & 0.7 & 1.1 \\
Merck & 11.1 & $169^{*}$ & 30.3 & 12.0 & 571 & 505 & 93.2 & 4.5 & 2.4 \\
STG1 & 5.9 & $250^{*}$ & $43.0^{*}$ & 34.6 & 576 & 524 & 0.2 & 96.8 & 2.9 \\
P25 & 57.4 & $25^{\mathrm{A}}$, & 17.0 & 19.8 & 553 & 595 & 81.7 & 15.4 & 3.0 \\
& & $40^{\mathrm{R}}$ & & & & & & & \\
TIO10 & 334.8 & $15^{*}$ & 9.4 & 2.5 & 559 & & 86.2 & 0 & 13.8 \\
\hline
\end{tabular}

* values determined and reported previously [12, 39] 
Graphical Abstract

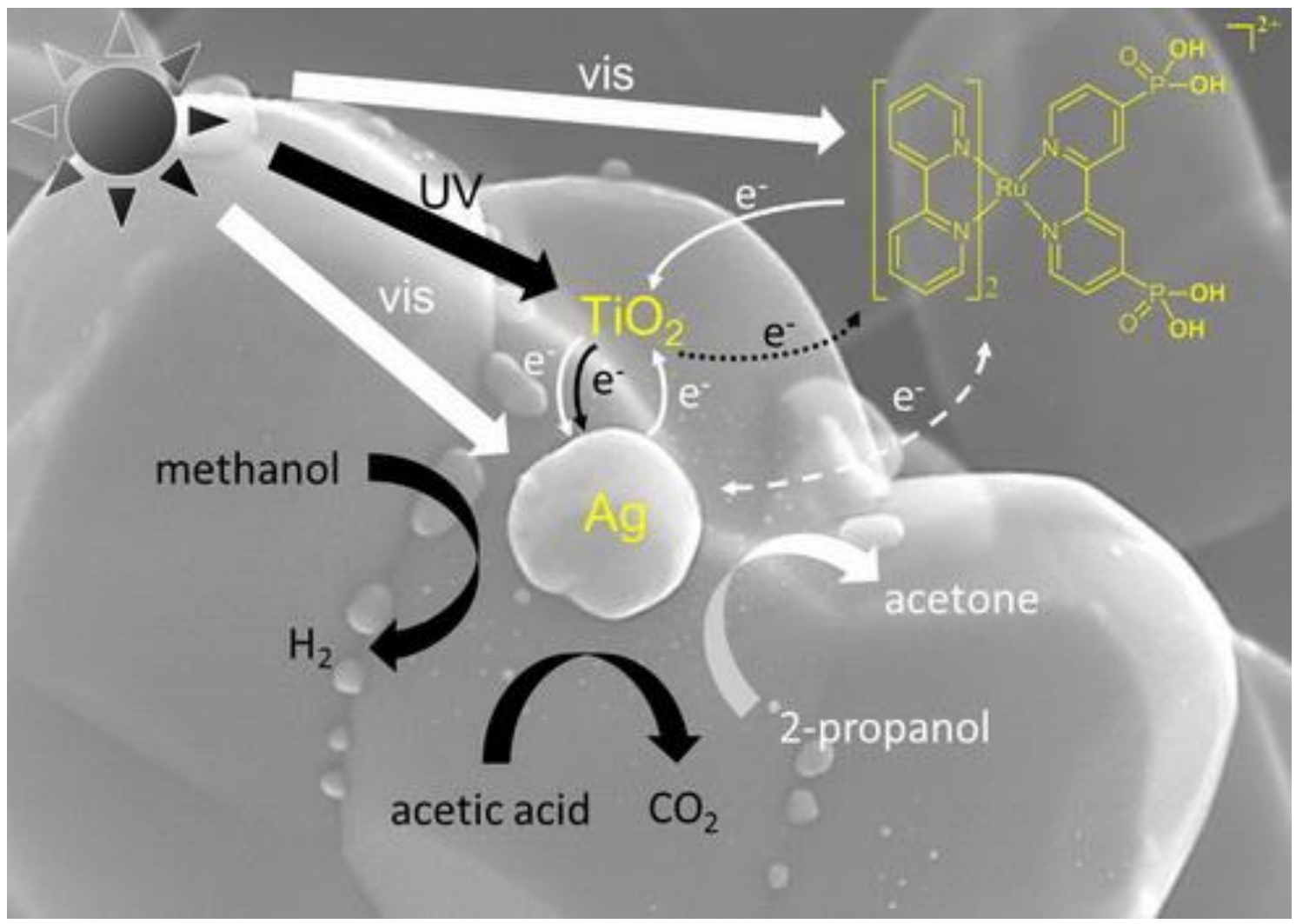

